Archives

$26 \mid 2001$

Du cahier de la coutume ... au livre // Études urbaines

\title{
Les éditions des coutumes d'auvergne
}

Anne Zink

\section{(2) OpenEdition}

Journals

\section{Édition électronique}

URL : http://journals.openedition.org/ccrh/1513

DOI : $10.4000 /$ ccrh. 1513

ISSN : $1760-7906$

Éditeur

Centre de recherches historiques - EHESS

Édition imprimée

Date de publication : 20 avril 2001

ISSN : 0990-9141

\section{Référence électronique}

Anne Zink, "Les éditions des coutumes d'auvergne », Les Cahiers du Centre de Recherches Historiques [En ligne], 26 | 2001, mis en ligne le 30 novembre 2008, consulté le 19 avril 2019. URL : http:// journals.openedition.org/ccrh/1513; DOI : 10.4000/ccrh.1513

Ce document a été généré automatiquement le 19 avril 2019.

Article L.111-1 du Code de la propriété intellectuelle. 


\title{
Les éditions des coutumes d'auvergne
}

\author{
Anne Zink
}

La coutume d'Auvergne a été rédigée en 1510 et bien que l'Auvergne soit située dans le ressort du parlement de Paris, sa coutume n'a pas été réformée ultérieurement. La région Auvergne actuelle regroupe quatre départements : la Haute-Loire constituée dans sa plus grande partie du Velay qui à l'époque moderne faisait partie du Languedoc, l'Allier qui correspond pour l'essentiel à l'ancien Bourbonnais, le Puy-de-Dôme et le Cantal; l'Auvergne d'Ancien Régime et sa coutume ne s'étendaient, par contre, pour l'essentiel, que sur ces deux derniers départements et sur la région de Brioude qui fut rattachée à la Haute-Loire. Alors que, dans le Bassin parisien, les coutumes ont été rédigées dans le cadre judiciaire et administratif de la juridiction intermédiaire, sénéchaussée ou bailliage, celui de la coutume d'Auvergne la rapproche des coutumes de la périphérie rédigées dans le cadre d'une province plus ou moins étendue: celles du Nivernais et de la Touraine correspondent approximativement à un département, la Normandie et la Bretagne sont sensiblement plus grandes, le Poitou, la Bourgogne ou l'Auvergne se situent dans l'intervalle. Alors que le nombre moyen d'articles dans les coutumes générales s'établit à 268, la coutume d'Auvergne en compte 583; mais elle n'est pas isolée: la coutume du Bourbonnais en dénombre 555, celle du Nivernais 611 , celle de Normandie 623 et celle de Bretagne 739'.

2 Si l'on en croit la Bibliographie des coutumes d'André Gouron et Odile Terrin ${ }^{2}$, l'Auvergne se situerait, de ce point de vue, aussi, dans le groupe de tête: si la Normandie et Paris figurent dans ce recueil avec plus de deux cents entrées et la Bretagne avec cent soixantequatre, les coutumes suivantes ont moins de cent entrées : trois d'entre elles seulement précèdent l'Auvergne qui, sous ce critère, semble aussi occuper une place un peu exceptionnelle. En réalité, il faut revoir cette place à la baisse : en effet, si sous la rubrique de chaque province, les auteurs du répertoire ont logiquement fait figurer à côté des coutumes et de leurs commentaires, des recueils d'arrêts et des traités de droit qui ne concernaient qu'un seul point des coutumes, dans certains cas, ils ont également pris en compte toute l'œuvre d'un légiste, sous prétexte que celui ci avait travaillé dans un de ses 
livres sur la coutume en question. C'est ce qui a eu lieu dans le cas de l'Auvergne : si le livre de Jean Masuer appelé Le Masuer en français ${ }^{3}$ concerne bien la coutume d'Auvergne dont ce légiste a donné au public une version antérieure de cinq ans à la rédaction officielle, version qui pour cette raison n'a jamais été rééditée, le reste de son œuvre consiste dans les vingt-cinq éditions tant latines que françaises de sa Pratique $e^{4}$ qui ne porte pas spécialement sur le droit auvergnat et qui a toujours été éditée hors de la province $^{5}$. Si on écarte cet ouvrage, on tombe à trente entrées, trente-deux si on ajoute les deux éditions qui ne figurent pas dans la Bibliographie de Gouron. L'Auvergne rentre alors dans le rang : elle appartient au groupe des treize coutumes générales qui ont donné lieu à la publication de vingt à cinquante ouvrages imprimés et qui précèdent les dix-neuf autres qui en ont eu de dix à dix-neuf. Même si l'Auvergne représente en général dans les cartes de l'édition une zone de faible densité, elle a eu ses imprimeurs ${ }^{6}$ et elle n'est pas aussi enclavée qu'on pourrait le croire : Lyon n'est pas loin et, par la Limagne, Riom et Clermont-Ferrand ${ }^{7}$ entrent sans peine en contact avec Paris et les villes de la Loire. Entre les vingt coutumes générales qui n'ont eu aucun commentaire imprimé, les trente et une qui en ont eu moins de cinq, l'Auvergne avec ses six commentateurs appartient au groupe déjà favorisé des onze coutumes qui en ont eu de cinq à neuf, derrière la Bretagne, Paris, la Bourgogne et la Normandie qui en ont eu de treize à dix-neuf.

L'Auvergne est également représentative dans la mesure où on a, dans un premier temps, beaucoup d'éditions de la coutume seule et peu de livres dans lesquels elle est accompagnée d'un commentaire ${ }^{8}$, alors qu'ultérieurement, on édite presque toujours la coutume commentées.

La coutume d'Auvergne est donc une grosse coutume avec un ressort relativement étendu et un nombre d'éditions et de commentateurs qui la situent dans la bonne moyenne alors que la province n'a jamais été particulièrement riche en ateliers d'imprimeurs et qu'elle dépend du parlement de Paris. Son histoire éditoriale offre donc un bon exemple pour s'interroger sur le nombre et l'évolution dans le temps de ces éditions, les débuts de l'imprimerie en province et la stratégie des libraires-éditeurs, l'intérêt des commentaires qui accompagnent la coutume à partir de la fin $d u x{ }^{e}$ siècle et l'apport de ces publications à la culture locale.

\section{Chronologie des éditions de la coutume}

5 Le 3 août 1510, à la fin de l'assemblée des états convoquée pour rédiger la coutume d'Auvergne, les commissaires du roi la publièrent et la firent signer par un certain nombre des députés, puis ils ordonnèrent «que six Registres ou Livres Coutumiers contenans lesdits Coutumes générales et particulières seront faits et écrits... l'un desquels sera mis au greffe... du parlement ", quatre pour les sièges des bailliages et sénéchaussées de la province afin que les plaideurs ayant à faire preuve de la coutume puissent en obtenir des extraits et le dernier, à la requête du pays d'Auvergne, sera déposé dans ses archives. Un de ces exemplaires manuscrits est conservé à la bibliothèque municipale de Clermont-Ferrand ${ }^{10}$.

6 Ce sont ces manuscrits que les éditions imprimées de la coutume ont suivis. Ces éditions comportent parfois des coquilles, des petites lacunes et mêmes des inversions mais il s'agit d'infidélités involontaires. Que les éditions se contentent de reproduire le texte de 
la coutume ou qu'elles intercalent les gloses d'un commentateur, elles se veulent fidèles à la lettre du texte officiel alors que leur présentation matérielle peut beaucoup varier.

7 Jusqu'au paragraphe précédent, je parlais de la coutume d'Auvergne au singulier parce que je cherchais à évaluer la représentativité de l'entité que la coutume d'Auvergne représentait dans l'ensemble du corpus formé par les coutumes des différents ressorts du royaume. À partir de maintenant, je distinguerai «la coutume générale» et «les coutumes locales », mais je dirai « les coutumes » au pluriel pour désigner l'ensemble qui a été rédigé par la même assemblée, lors d'une seule session, et qui a fait l'objet d'une seule publication et d'un seul procès-verbal. En employant le pluriel, je me conforme, de plus, au titre qu'ont adopté presque toutes les éditions. Le manuscrit original commence, sans titre général, par celui du premier chapitre ; on peut supposer qu'une page initiale a été perdue mais on peut aussi penser que la forme originale a bien été celle-là car les éditions imprimées commencent elles aussi toujours abruptement par les mots «Des adjournemens », titre du premier chapitre ${ }^{11}$. En revanche Louis XII dans ses Lettres de commission emploie toujours le pluriel quand il demande de rédiger « lesdites coustumes desdits bas et hauts Païs, tant générales que locales ». Le pluriel utilisé dans le titre des éditions imprimées a pu venir de là.

Pour dresser la liste des éditions imprimées des coutumes d'Auvergne, j'ai mis en œuvre, les ressources combinées de la Bibliographie des coutumes ${ }^{12}$, du Répertoire des livres imprimés en France ${ }^{13}$, et de la BMIU de Clermont-Ferrand. Dans l'état actuel de mes investigations, cette liste dans laquelle figurent toutes les éditions des coutumes données dans leur entier ou en partie, seules ou accompagnées d'un commentaire et/ou d'instruments de consultation, comprend vingt-neuf entrées. Je la donne sous la forme de notices qui rappellent beaucoup la Bibliographie des coutumes de laquelle certaines sont directement reprises mais, d'une part pour les raisons que j'ai exposées plus haut, j'en ai exclu les ouvrages de Masuer, les uns parce qu'ils ne concernent pas spécialement l'Auvergne, l'autre parce que la version qu'il donne de la coutume est antérieure à la rédaction officielle ${ }^{14}$; d'autre part, dans le souci de mettre en lumière l'activité éditoriale, j'ai rangé ces notices dans l'ordre chronologique.

Tableau no $\underline{1}^{15}$

$\mathbf{n}^{\circ}$ 1. Les coustumes du hault \& bas pays Dauvergne. A vendre à Paris rue Saint-Jacques, à Lyon rue Mercière et à Clermont chez Loys Maritain libraire. Imprimées à Paris le 20 mai 1511 pour Jean Petit. (17,5 x 12,2). Avec privilège.

$\mathbf{n}^{\circ}$ 2. Les coustumes du hault et bas pays Dauvergne. A vendre à Paris rue Saint-Jacques à la fleur de lys d'or, à Lyon rue Mercière et à Clermont chez Loys Maritain libraire. Imprimées à Paris le 31 septembre 1511 pour Jean Petit. (14,5 x 10,2). Avec privilège.

$11 \mathbf{n}^{\circ}$ 3. Les grandes coustumes generalles du hault et bas pays dauvergne nouvelleme(n)t corrigees avec plusieurs additions sur certains articles et ung extraict du proces verbal sur le dernier article des apchas (sic) lotz et ve(n)tes et sur plusieurs aultres articles contenues audict extraict : que devant navoit este imprime audict coustumier. À vendre à Lyon chez Jacques Maréchal près Notre-Dame de Confort, et à Clermont en la boutique dudit Maréchal devant Notre-Dame de Grâce. Imprimées à Clermont par Jacques Maréchal imprimeur et libraire devant Notre-Dame de Grâce. 30 avril 1523. In- $8^{\circ}$. Avec privilège

$12 \mathbf{n}^{\circ}$ 4. Les coustvmes du hault et bas pays Dauvergne nouvellement imprimees veues \& corrigees. À vendre à Lyon rue Mercière en la boutique Nicolas Petit et à Clermont à l'imprimerie, 1538. $(16,6$ x 10,1). 
$\mathbf{n}^{\circ} \mathbf{4}$ bis. Les coustvmes du hault et bas pays Dauvergne nouuellement imprimees veues \& corrigees. 1538. À vendre à Clermont en la boutique de Jean Durand devant Notre-Dame de Grâce. Imprimées à Clermont par Nicolas Petit, 28 mars 1538. $(16,6$ x 10,1).

$\mathbf{n}^{\circ}$ 5. Sensuyt le titre d'assiette de rente, auquel consequemment sont adjoustees les coustumes locales du hault et bas pays d'Auvergne: ensemble les noms des villes, chastellenyes, seigneuries et juridictions. Lyon, Antoine Vincent, 1548. In- ${ }^{\circ}$.

$\mathbf{n}^{\mathbf{0}}$ 6. Bessian de Preyssat, Arvernorvm consvetvdines... annotationes, Lyon, Antoine Vincent, 1548. (16,7 x 10,2). Avec privilège.

$\mathbf{n}^{\mathbf{0}}$ 7. Aymon, Commentarii in consuetudines Arverniae, Paris, Poncet Le Preux, 1548. In-fo

$\mathbf{n}^{\circ}$ 8. Aymon, Commentarii in consuetudines Arverniae, Paris, Poncet Le Preux, 1549. (32 x 21,3). Avec privilège.

$\mathbf{n}^{\circ}$ 9. Les Coustumes du Hault et Bas pays dauvergne nouvellement Imprimees, revues, corrigees oultre les precedentes Impressions. Plus de nouveau y est adiouste le Train de practique. À Clermont, Jean Durand près Notre-Dame de Grâce. 1552. (11,3 x 7,5).

$\mathbf{n}^{\mathbf{o}}$ 10. Les Coustumes du Hault et bas païs d'Auvergne, revues et corrigées avec le Train de practique. À Clermont, Jean Durand, 1570. In- $8^{\circ}$.

$\mathbf{n}^{\mathbf{o}}$ 11. Les Coustumes du Havt et Bas Pays Davvergne. Imprimé à Lyon chez Guichard Iullieron. 1581. (11,5 x 7,2).

$21 \mathbf{n}^{\mathbf{0}} \mathbf{1 2}$. Les coustumes du haut et bas pays d'Auvergne, nouvellement imprimees, revues \& corrigees oultre les precedentes impressions. Clermont, imprimerie Jean Durand pres Notre Dame de Grâce, 1587. (16,8 x 11,2). Deux exemplaires à la BMIU.

$\mathbf{n}^{\mathbf{0}}$ 13. Basmaison-Pougnet (Jean), Paraphrases de M. Iean de Basmaison Pougnet advocat en la seneschaulcee et siège présidial de Riom, sur les coutumes du bas et hault pays d'Auvergne, avec les annotations de M. Charles du Moulin et Procez verbal au long prins sur l'original desdictes coustumes. Clermont, Jean Durand, 1596. In- $4^{\circ}$.

no 14. Basmaison-Pougnet (Jean), Paraphrases de M. de Basmaison Pougnet... sur les coutumes du bas et hault pays d'Auvergne, avec les annotations de M. Charles Du Moulin, Clairmont, Bertrand Durand, 1608. (16,0 x 12,0).

$\mathbf{n}^{\mathbf{o}}$ 15. Rigault (Antoine) De diversis temporibus et terminis legis municipalis Avernorum cum iure civili, canonico, Gallico, consuetudinario collatis: cumque analatyca... Rossillonensis edicti..., Paris, François Huby, 1613. (17,2 x 11,3). Avec Privilège.

no 16. Basmaison-Pougnet (Jean), Paraphrases de M. Jean de Basmaison... sur les coutumes du bas et hault pays d'Auvergne, avec les annotations de M. Charles Du Moulin, Clairmont, Bertrand Durand, 1618. In $-4^{\circ}$. Avec privilège.

$\mathbf{n}^{\mathbf{0}}$ 17. Les coustumes du bas et haut pays d'auvergne et les procez verbaux au long... nouvellement imprimees, reveues \& corrigees oultre les precedentes impressions... augmentees d'une table alphabetique des coutumes locales... plus a été adjouté les règlements de la justice...; ensemble les taxes des expéditions qui se font en icelles. Clairmont, Bertrand Durand 1627. (15,9 x 9,6).

$\mathbf{n}^{\mathbf{o}}$ 18. Les coustumes locales du bas et hault Pays d'Auuergne. Et le Procès Verbal au long desdictes Coustumes. Clairmont, Bertrand Durand, 1627. (22,3 x 16,1).

$\mathbf{n}^{\mathbf{0}}$ 19. Basmaison-Pougnet (Jean), Paraphrases sur les coutumes du bas et hault pays d'Auvergne par M. Jean de Basmaison Pougnet, ...avec les annotations de $\mathrm{M}^{\mathrm{e}}$ Charles du 
Moulin advocat au parlement et deux tables alphabétiques l'une sur les coutumes locales, et l'autre des matières principales contenues ez articles et paraphrases desdictes coutumes. Clairmont, Bertrand Durand, 1628. (22,3 x 16,1).

$\mathbf{n}^{\mathbf{0}}$ 20. Basmaison-Pougnet (Jean), Paraphrases de M. de Basmaison Pougnet... sur les coutumes du bas et hault pays d'Auvergne, Clermont, Bertrand Durand, 1638. In- $4^{\circ}$.

$\mathbf{n}^{\circ}$ 21. Aymon, Bessian et Durand (George), Coustumes d'Auvergne paraphrasées par Aymon et Bessian. Traduicts du latin et enrichis d'observations, par $\mathrm{M}^{\mathrm{e}}$ George Durand avocat au parlement. Clermont, Bertrand Durand, $1640 .(23,5 \times 17,2)$. Trois exemplaires à la BMIU.

$\mathbf{n}^{\circ}$ 22. Bessian de Preyssat (Jean), Annotationes in consuetudines Arvernorum quibus in fine accessit copiosus rerum et verborum Index. Editio postrema castigiator. Trajecti ad Rhenum, G. Boschman, 1661. (15,5 x 9,5).

$\mathbf{n}^{\mathbf{0}}$ 23. Basmaison-Pougnet (Jean) et Consul (Guillaume) Coustumes du haut et bas pays d'Auvergne avec la Paraphrase de M. Jean de Basmaison Pougnet... et les notes de M. Charles du Moulin et le procès-verbal en long... Revue et beaucoup augmentée par $\mathrm{M}^{\mathrm{e}}$ Guillaume Consul, advocat en parlement, Clermont, Jean Barbier, 1667. (23 x 17,6). Avec privilège.

$\mathbf{n}^{\circ}$ 24. Les coustumes locales du bas et hault Pays d'Auuergne. Et le Procès Verbal au long desdictes coustumes. Corrigées... par Maître G. Consul... Clermont, Iean Barbier, proche Notre Dame de Grâce, 1667. (23,7 x 17,6). Avec privilège.

$\mathbf{n}^{\mathbf{0}}$ 25. Prohet (Claude-Ignace), Les coutumes du haut et bas païs d'Auvergne conférées avec le droit civil et avec la coutume de Paris, de Bourbonnais, de la Marche, de Berri et de Nivernais... des observations sur les coutumes générales et locales de la même province d'Auvergne : l'Histoire de ce qu'il y a de plus considérable dans chaque lieu, par les Fondations des Eglises, par les Personnes illustres originaires de cette Province, par les familles qui ont possédé les plus grandes terres et en quelles sénéchaussées et bailliages les lieux particuliers ressortissent. Par Claude Ignace Prohet, Paris, J.-B. Coignard, 1695. $(22,3 \times 16,6)$.

$5 \mathbf{n}^{\circ}$ 26. Texte de la coutume du Haut et Bas Pays d'Auvergne. Imprimé sur un manuscrit original dont on a suivi l'Orthographe. Clermont-Ferrand, P. Viallanes, 1740. In-12.

$\mathbf{n}^{\mathbf{0}}$ 27. Prohet (Claude-Ignace), Artaud, Coutumes du Haut et Bas Pays d'Auvergne avec ... les observations de $\mathrm{M}^{\mathrm{e}}$ Claude-Ignace Prohet... augmentée de nouvelles notes dont les principales sont sur les articles de la coutume qui ont été abrogés ou changés par les nouvelles ordonnances du roi Louis XV par Mr. ${ }^{* * *}$ (P.-P Artaud) avocat en parlement, Clermont-Ferrand, avec un recueil des arrêts de la cour des Grands jours, Pierre Viallanes près les RR. PP. Jésuites, 1745. 21,2 x 14,0.

$\mathbf{n}^{\circ}$ 28. Prohet (Claude-Ignace), $\mathrm{M}^{\mathrm{e}}$ (P.-P. Artaud), MM. Toussaint etc. Coutumes observations de $\mathrm{M}^{\mathrm{e}}$ Claude-Ignace Prohet, et des explications... suivant les niveaux édits, déclarations et arrêts par $\mathrm{M}^{\mathrm{e}}{ }^{* * *}$ (P.-P. Artaud) avocat en parlement (et avec les soins de $\mathrm{M}^{\mathrm{e}}$ Jean-François Gaultier-Biauzat, avocat)... revue... et augmentée des notes de MM. Toussaint Chauvelin, Julien Brodeau, Jean-Marie Ricard et autres célèbres jurisconsultes ; d'un sommaire en marge de chaque article, et une liste alphabétique des lieux..., Clermont-Ferrand, Pierre Viallanes, près l'ancien marché au blé, $1770 .(24,8$ x 18,3). Avec privilège. 
$\mathbf{n}^{\circ}$ 29. Chabrol (Guillaume-Michel), Coutumes générales et locales de la Province d'Auvergne avec les notes de Mes Charles du Moulin Toussaint Chauvelain, Julien Brodeau et Jean-Marie Ricard; des observations sur ces coutumes et sur le droit écrit qui régit une partie de la province et des notes historique sur les coutumes locales ; précédées de deux dissertations : l'une sur l'origine... de la diversité des Loix qui régissent cette province ; l'autre sur la forme dans laquelle la justice y a été administrée depuis l'origine de la monarchie par M. Chabrol, conseiller d'Etat, Riom, Martin Dégoutte, imprimeur-libraire, vis-à-vis de la fontaine des Lignes, 4 tomes, 1784-1787. In- $4^{\circ}$. CXCVIII+521, 899, 685+79, XLVII $+857+20+40$ ppnc.

$\underline{\text { Tableau n }} \underline{\underline{0}}$

Les éditions des coutumes d'Auvergne

\begin{tabular}{|c|c|c|c|c|c|}
\hline $\begin{array}{l}\text { Siliècles } \\
\text { Cownumes sentles: }\end{array}$ & $x V I^{18}$ & XVIII & XVITII" & $1511-1628$ & $1.6 .30 \cdots-11789$ \\
\hline Inaprinnéxes à Clernaomt & 5 & 3 & 1. & 7 & 2 \\
\hline Vejadues à Clermont & 2 & 0 & 0 & 2 & 0 \\
\hline Ailleurs & 2 & 0 & 0 & 2 & 0 \\
\hline Towlan! & 9 & 3 & $I$ & $n$ & 2 \\
\hline \multicolumn{6}{|l|}{ Countumess connmentióes } \\
\hline Inaprinées à Clernaont & 1 & 6 & 3 & 4 & 6 \\
\hline Vendues à Clermont & 0 & 0 & 0 & 0 & 0 \\
\hline Ailleurs & 3 & 3 & 0 & 4 & 2 \\
\hline Towal & 4 & 9) & 3 & 8 & 8 \\
\hline TOTAL & 1.3 & 12 & fif & 19 & 10 \\
\hline
\end{tabular}

Livres vendus ou innprimes à Clermont

ND

Le tableau $\mathrm{n}^{\circ} 2$ regroupe les données du tableau $\mathrm{n}^{\circ} 1$, en y ajoutant, à titre de comparaison, le nombre total des livres parus à Clermont au XVI ${ }^{e}$ et au XVII ${ }^{e}$ siècle. La coupure par siècle n'est pas plus arbitraire qu'une autre, puisque, de toute façon, les besoins et les usages ne se modifient jamais tous en même temps. Dans le cas présent, elle est même heureuse car, comme nous le verrons, c'est entre 1587 et 1597 pour le fond, entre 1570, 1587 et 1608 pour la forme, qu'on peut situer, en ce qui concerne les éditions des coutumes d'Auvergne, la transition entre le $\mathrm{XVI}^{\mathrm{e}}$ et le $\mathrm{xVII}{ }^{\mathrm{e}}$ siècle. À partir de cette dernière date, on peut considérer que les imprimeurs clermontois ont rejoint leurs confrères lyonnais et parisiens dans l'adoption des normes de présentation qui resteront classiques.

En revanche si on veut tenir compte de leur nombre, c'est après 1628 qu'il faut mettre la coupure : presque deux fois plus de livres contenant les coutumes pendant les cent dixsept premières années que pendant les cent cinquante-neuf années suivantes; c'est pourquoi les deux dernières colonnes du tableau adoptent cette coupure. Même si le Répertoire des livres imprimés en France au dix-huitième siècle ne couvre pas encore l'Auvergne et même si toutes les provinces sont en perte de vitesse par rapport à Paris, il est frappant de voir les éditions des coutumes s'effondrer pendant ce siècle alors que le nombre de livres édités s'envole dans le royaume. Au XvI ${ }^{\mathrm{e}}$ siècle, plus d'un livre sur quatre mis en vente ou imprimé à Clermont est un coutumier; au XVII ${ }^{e}$ siècle, on tombe à un sur dix-neuf. Ces livres, en revanche, sont désormais plus volumineux : en effet, alors qu'au $\mathrm{XVI}^{\mathrm{e}}$ siècle neuf coutumes sur treize sont imprimées sans commentaire, elles ne 
sont plus que quatre sur seize soit un quart pour l'ensemble des XVII et XVIII siècles, deux sur dix soit un cinquième après 1630.

\section{L'imprimerie à Clermont-Ferrand au XVI siècle}

\section{Tableau et annexe}

41 Dans le tableau $\mathrm{n}^{\circ} 1$, je n'ai donné que le titre proprement dit, coupé parfois de points de suspension quand les indications données reprennent mot pour mot celles d'une édition précédente ou deviennent trop prolixes au XVIII ${ }^{\mathrm{e}}$ siècle. En ce qui concerne les lieux d'édition et de vente, les éditeurs, les libraires et la date, j'ai gardé toutes les indications utiles à mon propos mais je ne pouvais pas rendre compte de l'apparence des livres dont l'évolution caractérise le $\mathrm{XVI}^{\mathrm{e}}$ siècle car de telles indications occupent trop de place. C'est pourquoi j'ai complété le tableau par une annexe qu'on trouvera à la fin de l'article.

Celle-ci décrit jusqu'en 1608 l'allure matérielle des éditions que recense le tableau $n^{\circ} 1$. Elle prend modèle sur le Répertoire des livres imprimés au XVI siècle, en essayant de mieux rendre compte de l'utilisation des majuscules dans les titres de livres. À partir de 1587, l'atelier clermontois semble aussi bien équipé que les ateliers lyonnais et parisiens; si l'annexe continue jusqu'en 1608 , c'est pour confirmer le niveau atteint et pour donner une idée des éditions ultérieures. Au-delà, il ne m'était plus utile de prêter autant d'attention à la présentation matérielle des livres. Dans cette annexe, je cherche à décrire la façon dont se présente chaque édition : format, typographie, mise en page, présence de fleurons, de frises, de culs-de-lampe ou de marques d'éditeur, de façon à suivre le travail des imprimeurs, éditeurs, auteurs et libraires, à comprendre peut-être l'attente du public et à faire profiter le lecteur de la documentation disponible à la BMIU de Clermont où j'ai pu consulter un exemplaire de plus des deux tiers de ces éditions ${ }^{16}$.

\section{L'objet-livre}

Sur les quatorze éditions cataloguées jusqu'en 1608, la moitié ont été imprimées à Clermont, quatre à Paris et trois à Lyon. Avec l'aide de l'annexe, on voit se succéder les modifications attendues dans la présentation des livres et émerger des conventions auxquelles tous les imprimeurs se rallient à peu d'années de distance.

Les petits formats sont de beaucoup les plus nombreux : deux éditions in-folio à Paris au milieu du siècle ( $n^{\circ} 7$ et 8$)$, trois in- $12^{\circ}$, deux à Clermont, l'autre à Lyon $\left(n^{\circ} 9,11\right.$ et près de deux siècles plus tard le $\mathrm{n}^{\circ} 20$ ), trois in $-4^{\circ}$ au tournant des XVI $I^{\mathrm{e}}$ et XVII ${ }^{\mathrm{e}}$ siècles à Clermont $\left(n^{\circ} 13,18\right.$ et 19$)$, les six autres de toutes dates et de toutes origines in- $8^{\circ}$. Les éditions $n^{\circ} 1$, 2 et 3 (1511-1523) à Paris ou à Clermont emploient des caractères gothiques. Les caractères romains apparaissent avec le $n^{\circ} 4$ (1538) à Clermont et l'emportent en 1548-1549 dans les éditions $\mathrm{n}^{\circ} 6$ et 8 à Lyon et à Paris ; en 1552 à Clermont également, on ne trouve plus qu'eux ( $\left.n^{\circ} 9\right)$ mais alors que dans les deux grands centres, quatre ans plus tôt, les éditeurs disposent de caractères romains et italiques, majuscules et minuscules de toutes tailles, l'éditeur de Clermont ne travaille encore qu'avec des caractères romains sans variété. Une génération plus tard, Clermont dispose à son tour de caractères nombreux et variés $\left(\mathrm{n}^{\circ} 12,1587\right)$ mais moins que Lyon, qui a encore élargi sa palette $\left(\mathrm{n}^{\circ}\right.$ $11,1581)$. C'est en 1627 ( $n^{\circ} 17$ et 18 ) que le retard est rattrapé. 
Jusqu'à l'édition $\mathrm{n}^{\circ} 4$, celle de 1538 , on ne compte que les feuillets; la pagination commence en 1548-1549 à Paris et à Lyon et gagne Clermont en 1552, mais, d'une part, dans l'édition de 1552 et dans celle de 1587, il y a une erreur, si bien qu'à partir de la page 53 dans l'une, 119 dans l'autre, les pages de droite se retrouvent paires; une erreur du même type intervient encore dans l'édition $\mathrm{n}^{\circ} 12$ en 1587 ; d'autre part, le Train de pratiques qui suit les coutumes est encore folioté. En 1570 dans l'édition $\mathrm{n}^{\circ} 10$, la table alphabétique des coutumes locales n'est ni paginée ni foliotée.

Le nom de l'auteur des commentaires, ou des corrections ( $\left.n^{\circ} 19\right)$, ne se détache pas, il est toujours contenu dans le titre. L'invitation à acheter sous la forme de l'expression « À vendre à tel endroit » ne figure que dans les cinq premières éditions mais quand l'éditeur donne son adresse, en page de garde, à la suite de son nom précédé de « chez » ou de " apud", le sens est bien le même; et il n'est pas très différent quand l'adresse n'est pas introduite par une de ces prépositions. Le colophon qui donnait parfois le jour et le mois d'impression, disparaît dès 1538 dans l'édition $\mathrm{n}^{\circ} 4$; il réapparaît curieusement en 1613 (n $\left.{ }^{\circ} 15\right)$. Il est remplacé en page de garde par la date, d'abord placée au-dessus du nom de l'éditeur, puis en dessous suivant l'usage qui a perduré : lieu, éditeur, date. Elle est donnée en chiffres arabes de 1538 à 1587 et au-delà en chiffres romains.

Cinq éditions affirment avoir reçu un privilège soit du parlement, soit du roi et $l^{\prime}$ impriment $\left(\mathrm{n}^{\circ} 1,2,3,8,16\right)$, trois autres le mentionnent sans le donner $\left(\mathrm{n}^{\circ} 6,13,19\right)$. Si les éditions $\mathrm{n}^{\circ} 1$ et 2 signalent si précisément leur date de parution, c'est peut-être parce que le privilège, deux ans pour l'une, trois ans pour l'autre, est de courte durée. Dans le privilège accordé pour l'édition $\mathrm{n}^{\circ} 3$ par le roi, celui-ci dit avoir pris en considération les grands frais engagés par Jacques Maréchal pour faire " visiter et corriger les coustumes ». Un siècle et demi plus tard Jean Barbier, alors qu'il sollicite un privilège pour une réédition du commentaire de Jean de Basmaison-Pougnet augmenté par Guillaume Consul, souligne l'intérêt économique de cette protection en disant qu'«il craint que d'autres ne voulussent faire le semblable, ce qui lui serait un grand préjudice ». Jusque-là les éditions purement clermontoises étaient parues sans privilège : les éditions $\mathrm{n}^{0} 3$ et 4 qui en avaient sollicités au Xvi ${ }^{\mathrm{e}}$ siècle, avaient des liens avec Lyon.

8 Presque toutes les éditions comportent une table des chapitres de la coutume générale. En 1552, l'éditeur Jean Durand publie à la suite des coutumes un Train de pratique qui comporte une excellente table des matières, mais pour les coutumes, il faut attendre l'édition lyonnaise de Julliéron en 1570 ( $\left.\mathrm{n}^{\circ} 11\right)$ pour en disposer ; ultérieurement, on en trouve une dans l'édition $\mathrm{n}^{\circ} 14$ de Bertrand Durand en 1627 et une très complète dans l'édition $n^{\circ} 19$ de Jean Barbier en 1667. À partir de 1608, les éditions des coutumes locales sont accompagnées d'une table alphabétique des localités $\left(\mathrm{n}^{\circ} 14,17,18\right)$ qui contient 679 entrées pour la basse Auvergne et 252 pour la haute ${ }^{17}$.

Dans ce corpus, la page de garde en rouge et noir n'apparaît qu'au milieu du XVIII ${ }^{e}$ siècle. Les éditions parisiennes $\mathrm{n}^{\circ} 1,2$ et 8 portent en page de garde la marque de leur éditeur de même que l'édition $\mathrm{n}^{\circ} 3$ réalisée à Clermont pour un éditeur lyonnais et avec sa marque. L'édition $\mathrm{n}^{\circ} 4$, purement clermontoise, $\mathrm{n}$ 'a que deux petits fleurons sur une page de garde par ailleurs aérée et bien construite. La marque de l'éditeur clermontois Jean Durand apparait en 1587 dans l'édition $n^{\circ} 9$; elle est reprise par son fils dans l'édition $n^{\circ} 18$. C'est aussi à partir de 1587 que sortent des éditions soignées avec des culs-de-lampe et des frises de grotesques, d'oiseaux ou de motifs floraux. La marque des Durand représente une main sortant d'un nuage qui tient une aiguière et qui verse de l'eau sur une épée qu'une autre main tend dans les flammes d'un feu. Autour est inscrit la devise «Durant 
temperata ». Guy de Tervarent donne l'explication de cette marque : l'eau versée d'une aiguière représente la tempérance qui adoucit les choses violentes: le feu et l'épée sur lesquelles elle est versée. La devise inspirée par le nom de l'éditeur et par l'aphorisme "violenta non durant", «les choses violentes ne durent pas", signifie «les choses modérées durent $»^{18}$.

\section{Éditeurs et éditions}

Si on ajoute au tableau $n^{\circ} 2$ qu'en 1518 le Manuale seu instructium curatorum, imprimé à Lyon à partir d'une édition parisienne corrigée et examinée par l'official de Clermont, est en vente "dans la boutique d'Antoine Calvet devant l'église Notre-Dame appelée en français de Grâce ${ }^{19}$, on a l'impression que la boutique de libraire, complétée en 1523 par un atelier d'imprimeur, est restée au même endroit, de 1518 à 1567, au moins «Place devant Clermont » c'est-à-dire près de la cathédrale ${ }^{20}$.

51 Si les coutumes d'Auvergne ont été imprimées moins d'un an après leur rédaction, c'est peut-être grâce à Antoine Duprat premier président du parlement de Paris, qui avait été commis à cette rédaction et qu'une dédicace compare pour cette raison à Justinien ${ }^{21}$. Quand le grand éditeur Jean Petit fait imprimer les coutumes d'Auvergne à Paris à deux reprises en 1511, il s'était déjà assuré d'un diffuseur à Clermont ${ }^{22}$. Les éditions $\mathrm{n}^{\circ} 3$ et 4 témoignent d'une collaboration entre Lyon et Clermont: la première en 1523 est imprimée à Clermont, avec sa marque, pour Jacques Maréchal, un éditeur lyonnais, lui aussi bien connu ${ }^{23}$; on peut lire sur la page de garde que le livre est en vente à Lyon et à Clermont même, dans cette boutique devenue également atelier ${ }^{24}$. Pour la seconde, imprimée également à Clermont en 1538 par Nicolas Petit, on a prévu deux pages de garde : sur l'une, on dit que le livre est en vente à Lyon dans la boutique de Nicolas Petit et à Clermont à l'imprimerie, sur l'autre, il n'est pas question de Lyon : à Clermont on le vend à la boutique de Jean Durand située à l'endroit habituel près de Notre-Dame de Grâce.

Les trois éditions parisiennes suivantes des coutumes, les $\mathrm{n}^{\circ} 7$ et 8 parus en 1548 et 1549 chez Poncet Le Preux et le $\mathrm{n}^{\circ} 15$ paru en 1613 chez François Huby, ne font pas état de rapports spécifiques avec Clermont. Nous verrons plus loin qu'il s'agit de commentateurs sans grand contact avec les réalités auvergnates. En 1548 également, un éditeur lyonnais Antoine Vincent, qui lui non plus n'indique pas de point de vente à Clermont, inaugure une formule qui sera reprise au XVII ${ }^{\mathrm{e}}$ siècle : éditer la coutume générale commentée $\left(\mathrm{n}^{\circ} 5\right)$ et les coutumes locales seules $\left(n^{\circ} 6\right)$ avec deux pages de garde, de façon à pouvoir vendre le tout en un ou en deux volumes ; le commentateur choisi est, de plus, mieux au courant de la jurisprudence locale. Une génération plus tard Guichard Julliéron imprimera à Lyon en 1581 une édition des coutumes honnête et modeste $\left(n^{\circ} 11\right)$ sans signaler lui non plus de liens avec Clermont.

53 Les Durand vont jouer un grand rôle dans l'édition clermontoise. Le Répertoire des livres imprimés n'en connaissait que deux : Jean et Bertrand. Pierre Eguillion montre qu'il y a eu successivement deux Jean qui ne sont pas faciles à distinguer: Jean I, 1530-1554, apparenté à Etienne et Antoine Durand, facteurs de Jacques Maréchal, et Jean II son fils 1560-1596. Les Jean vendent ou éditent dix-neuf livres au XVI ${ }^{e}$ siècle, dans quatre cas il s'agit des coutumes seules ou commentées ; Bertrand édite un livre au XVI et seize au XVII ${ }^{e}$ siècle, dont cinq coutumes seules ou commentées et un Train de Pratique prévu en 1652 pour être relié avec la coutume. Lors de la publication en 1628 des Paraphrases sur les 
coustumes de Jean de Basmaison-Pougnet $\left(\mathrm{n}^{\circ} 19\right)$, on voit que Bertrand Durand a un dépositaire à Paris. Il est remplacé par Jean Barbier : la traduction d'Aymon et Bessian par George Durand parue en $1640\left(n^{\circ} 21\right)$, porte en page de titre «par Bertrand Durand imprimeur du roy ", mais à la fin on lit «achevé d'imprimer par Jean Barbier ». Grâce à Pierre Eguillion nous voyons comment la transition s'est faite et que Bertrand Durand a pris place parmi les notables de la ville ${ }^{25}$.

C'est chez lui qu'a été publié le livre de Jean Savaron, Les Origines de Clairmont, ville capitale d'Auvergne en 1607, puis à nouveau en $1609^{26}$. Dans ce livre, Savaron, conformément aux normes historiographiques de l'époque, attribuait à Clermont des origines brillantes mais fabuleuses et faisait un grand usage de l'étymologie des noms propres. Écrire "Clairmont " pour rester plus près de l'orthographe latine n'était pas une erreur mais une fantaisie contraire à l'usage et destinée à donner du lustre à la ville. En 1699, l'édition des nouveaux statuts synodaux, le premier livre connu paru chez Bertrand Durand après la mort de son père, orthographiait «Clairmont » aussi bien le lieu d'édition que le nom du diocèse. Il en va de même en 1601 pour le Règlement de justice ordonné par les juges de la sénéchaussée et du présidial, cour dont Savaron était le président.

On peut rapprocher cette affectation de la façon dont Jean de Basmaison-Pougnet intervient d'une façon très personnelle en tête de son commentaire des coutumes. Je cite l'édition de $1608\left(\mathrm{n}^{\circ} 14\right)$ mais la préface qui est datée du $1^{\mathrm{er}}$ juin 1590 , figurait sans doute déjà dans la première édition connue, celle de $1596\left(n^{\circ} 13\right)$ : d'une part il dédie le livre "Aux Treze anciennes et maistresses villes du bas pays d'Auvergne", d'autre part il rappelle et les divisions récentes, et la délégation publique dont il avait été investi pour représenter la province aux deux sessions des états généraux tenus à Blois en 1576 et en 1588, et la députation auprès du roi dont il avait fait partie en $1578^{27}$. On est déjà là dans l'univers politique dont Savaron sera une grande figure, un régime dans lequel les magistrats, les cours de justice, les villes et les provinces ont des responsabilités qui s'expriment dans le cadre d'assemblées provinciales ou nationales. L'attachement à l'illustration de la petite patrie est une façon de conforter le poids des gouvernés les plus raisonnables, c'est-à-dire du tiers état, dans les conseils de la grande patrie. En 1640 encore $\left(n^{\circ} 20\right)$ l'éditeur Bertrand Durand et/ou l'avocat George Durand font écrire sur la page de garde deux devises : «qui multiplicat Iustitiam, multiplicat pacem » et «Averni latios se discere fratres Sanguine ab Iliaco populi » phrase qui faisait écho aux origines mythiques prêtées par Savaron à Clermont. Ce contexte fait penser que Bertrand Durand, simple échevin clermontois, n'a pas été à l'origine d'une orthographe choisie par des personnages d'envergure provinciale ou nationale mais il a pu s'y rallier très volontiers. Après 1629, alors que Savaron est mort en 1622, que le régime a repris à marche forcée son évolution vers l'absolutisme et que Bertrand Durand a cédé l'atelier à Jean Barbier, Clairmont redevient Clermont.

\section{Répondre aux besoins du public}

On peut penser que le choix de formats modestes correspond aux vœux du public. Au début du XVI ${ }^{\mathrm{e}}$ siècle, Jean Petit sortant deux éditions des coutumes à quatre mois l'une de l'autre, emploie pour la seconde des feuilles et des caractères plus petits et ses typographes font tout pour que le nombre de feuillets augmente le moins possible, par exemple en allant moins souvent à la ligne dans les titres. La deuxième édition est donc 
plus tassée, moins agréable à lire, moins coûteuse à expédier de Paris à Clermont et donc, sans doute, moins chère que la première.

Telle fut peut-être la politique des Durand au début des éditions de Basmaison-Pougnet. Bibliographie et Répertoire semblent d'accord pour voir dans l'édition de $1596\left(\mathrm{n}^{\circ} 13\right)$ un in- $4^{\circ}$ alors que celle de $1608\left(n^{\circ} 14\right)$ est un in- $8^{\circ}$. Après avoir satisfait une clientèle moins aisée, on serait revenu en $1618\left(\mathrm{n}^{\circ} 16\right)$ et en 1627 à un plus grand format. L'édition des coutumes de $1587\left(\mathrm{n}^{\circ} 12\right)$ prouve que dès cette date au moins, l'atelier de Jean Durand est équipé pour faire du travail soigné : mise en page aérée, polices de tous types et de toutes tailles, lettrines ornées, frises et culs-de-lampe ${ }^{28}$. Le livre a eu du succès puisque la BMIU en a deux exemplaires.

Les Coutumes accompagnées du Train de Pratique qui sont d'abord parues en in- $12^{\circ}\left(n^{\circ} 9\right)$ en 1552 seraient, si j'en crois les instruments de travail, parues en in- $8^{\circ}$ en $1570\left(n^{\circ} 10\right)$; mais j'hésite à les croire car selon la Bibliographie, l'édition de 1552 serait, elle aussi, in- $8^{\circ}$ alors que j'ai eu le volume entre les mains, que c'est un in- $12^{\circ}$ et que le Répertoire, lui aussi, le voit in- $12^{\circ}$. Ce n'est pas une édition soignée : le texte est très compact et il y a une erreur de pagination, mais c'est un livre qui a dû être utile car sa table des matières est très bien faite. Un bon livre de poche pour praticiens. Le seul ornement consiste à deux endroits en des vers de mirliton ${ }^{29}$. Dans l'exemplaire de la BMIU, les Coutumes et le Train de pratique étaient bien destinés à être reliés ensemble puisque la présence additionnelle du Train est annoncée sur la page de garde des Coutumes; mais les clients avaient la possibilité d'acheter les Coutumes seules accompagnées d'une autre page de garde, ou le Train de pratique seul puisqu'il a sa propre page de garde qu'on trouve à l'intérieur du volume conservé à la $\mathrm{BMIU}^{30}$. Cette édition ne sera reprise qu'un siècle plus tard, en 1667, par Jean Barbier ( $\left.n^{\circ} 19\right)$.

De telles options-pouvoir acheter un ouvrage seul ou en faire relier deux ensemble-sont également possibles entre la coutume générale commentée et les coutumes locales seules. On a vu plus haut que l'éditeur lyonnais Antoine Vincent avait proposé une formule de ce type en 1548 ( $n^{\circ} 5$ et 6 ) : d'un côté la coutume générale jusqu'au chapitre 30 commentée en latin par Bessian de Preyssat, de l'autre le chapitre $D^{\prime}$ assiette de rentes et les coutumes locales. L'édition $n^{\circ} 5$ ne comprend que la seconde partie, l'édition $\mathrm{n}^{\circ} 6$ comprend les deux. Le chapitre $31 D^{\prime}$ assiette de rentes est le trente et unième et dernier titre de la coutume générale, il comprend soixante dix-neuf articles. Pour Prohet en 1695, « c'est le règlement \& l'estimation pour asseoir et estimer les rentes mais comme le prix des choses a beaucoup augmenté, l'usage de ce titre est presque inutile».

60 En 1548 la hausse des prix n'est pas encore perçue comme irréversible et on juge bon de l'éditer avec les coutumes locales (annexe $\mathrm{n}^{\circ} 6$ ). Les deux textes posent en effet des problèmes de mémorisation: le chapitre 31 est plein de chiffres, chacune des coutumes locales est très courte, rarement plus de trois articles, ces articles répètent parfois les dispositions de la coutume générale, ils se ressemblent sans être les mêmes, il est impossible de retenir par cœur tant de coutumes, alors que les hommes de lois et même les simples particuliers finissent par connaître les grandes lignes de la coutume générale. C'est-à-dire qu'un procureur ou un avocat qui travaille pour des clients venant de différentes juridiction, a besoin, pour les conseiller, d'avoir sous la main la liste des coutumes locales. 
61 Dans les éditions de 1627-1628 ( $\left.n^{\circ} 17-19\right)$, le chapitre 31 reprend sa place dans la coutume générale; un jeu de pages de garde, d'allure semblable mais de contenu légèrement différent, doit permettre à l'acheteur de relier ensemble comme il l'entend la coutume générale seule ou commentée, les coutumes locales et le Train de pratique. La formule est reprise en 1667. En 1627 Bertrand Durand propose aussi en un volume, la coutume générale, les coutumes particulières, les règlements de justice et la taxe des expéditions dans un format beaucoup plus petit, 9,6 x 15,9. La présentation est soignée mais simple. Il revient ainsi à la même clientèle que celle qui était visée par le Train de pratique: des praticiens qui n'ont pas à résoudre des problèmes rares, mais qui ont besoin d'avoir différents types de renseignements dans un même manuel.

62 Alors que dans le premier tiers du XVII ${ }^{\mathrm{e}}$ siècle le nombre et la diversité des présentations mises à la disposition de la clientèle montrent que les coutumes sont un bon produit, on assiste ensuite à un espacement des éditions. Le fait que les éditions commentées l'emportent maintenant sur les éditions de la coutume seule, montre pourtant qu'il n'y a pas désaffection. Qu'attend-on, dans cette seconde période, de la coutume et de ses commentaires?

\section{Le temps des commentaires}

\section{Les commentateurs}

\section{Tableau no 3}

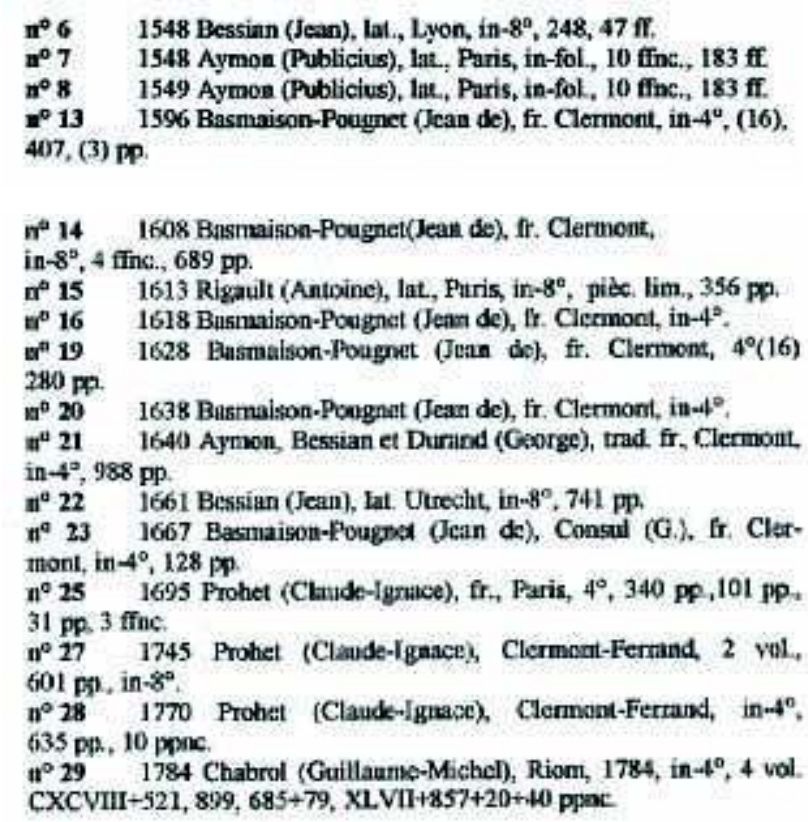

63 Le tableau $n^{\circ} 3$, tiré des mêmes sources et pour l'essentiel du tableau $n^{\circ} 1$ lui-même, montre que les juristes n'ont pas attendu le $\mathrm{XVII}^{\mathrm{e}}$ siècle pour faire paraître des commentaires des coutumes.

Deux commentateurs sont édités en 1548, l'un à Lyon, l'autre à Paris et ce dernier Aymon est immédiatement réédité à l'identique en 1549 ( $n^{\circ} 5$ et 6 ). Il est présenté par son éditeur comme piémontais, docteur en droit civil et en droit canon et président de Savoie. Il a été assigné à résidence sans savoir pourquoi pendant cinq ans à Montferrand, mais il connaît 
mal le droit auvergnat et s'y intéresse peu : il n'a pas jugé bon de reproduire dans son livre les coutumes locales et s'il cite des contemporains, ce sont toujours des Piémontais. Ses commentaires sont très courts, en postface il parle de glosellas. Il s'agit surtout pour lui de comparer le droit auvergnat à ses propres cours de droit. Prohet en 1695 ( $\left.\mathrm{n}^{\circ} 25\right)$ l'accuse de traiter de ces "matières sur les maximes des Ultramontains qui ne nous conviennent point : et c'est ce qui fait que ce commentaire n'est presque d'aucun usage ». C'est du droit auvergnat pour Parisiens, paraphrasé, de très loin, dans un latin fluide.

L'éditeur lyonnais présente Bessian ${ }^{31}$, comme un célèbre jurisconsulte qui fut avocat d'abord à Riom, puis à Toulouse. Le fait qu'il soit docteur en droit ne figure pas sur la page de garde, comme si l'éditeur tenait à le montrer en homme de terrain ; lui-même dit que son livre résulte de son expérience et il se donne réellement la peine d'expliquer à son lecteur certains termes de la coutume $\left(n^{\circ} 7\right)$. Prohet qui n'est pas indulgent avec ses prédécesseurs dit qu'il était difficile qu'il prît bien l'esprit de notre coutume à Toulouse où il s'était installé. Il est réédité à Utrecht en 1661 sans modification; en dépit du petit format, l'édition semble soignée mais la présence de caractères italiques ne fait pas longtemps illusion puisqu'ils sont employés sans tenir compte du sens du texte ( $\left.\mathrm{n}^{\circ} 22\right)$.

En $1613\left(n^{\circ} 15\right)$ Antoine Rigault fait paraître à Paris une comparaison entre les coutumes d'Auvergne, le droit civil, le droit canon et le droit coutumier français, avec une analyse de l'édit de Roussillon qui, cinquante ans plus tôt, avait fait commencer l'année en janvier et interdit aux magistrats de modifier les édits royaux, disposition qu'il était peut-être utile de rappeler en temps de régence. Antoine Rigault était avocat auprès de la sénéchaussée et du présidial de Riom, son oncle Gilbert Rigault était conseiller au présidial de Clermont. La dédicace parle des lois de la patrie en allitération avec l'oncle qui a tenu lieu de père, mais il ne semble pas y être aussi attaché que ceux qui éditent à Clermont chez Bertrand Durand, et les références en marge, à l'exception de Masuer, ne sont pas auvergnates; il a néanmoins le mérite de citer souvent les autres coutumes, le droit coutumier français en général et d'avoir réfléchi sur le rapport entre les coutumes et la législation royale.

En 1640 un avocat, George Durand fait paraître ce qu'il présente comme une traduction de Bessian et d'Aymon ( $\left.\mathrm{n}^{\circ} 21\right)$. En réalité, il apporte beaucoup plus qu'une simple traduction. Il a beaucoup élagué le texte de ses prédécesseurs et à la place il a rédigé des commentaires nouveaux plus proches de la pratique et de la jurisprudence auvergnates. Ses prédécesseurs, dit-il dans sa dédicace à un conseiller à la sénéchaussée, ont concilié « notre mère coutume » et « la loi » c'est-à-dire le droit romain, lui veut montrer que la coutume est affermie par les arrêts des cours souveraines, c'est-à-dire qu'il se situe du côté du droit français vivant. Il cite, souvent en les regroupant, les articles de la coutume qu'il fait suivre, sous les titres de Aymon puis de Bessian, des commentaires abrégés qu'il attribue à l'un puis à l'autre, puis sous le titre d'Addition il donne son propre commentaire. Le tout est suivi d'une table des matières très complète. La BMIU en possède trois exemplaires ce qui témoigne sans doute de sa diffusion.

Depuis un demi-siècle on disposait d'un autre commentaire des coutumes écrit en français et proche du terrain: celui de Jean de Basmaison qui allait faire l'objet de rééditions nombreuses et rapprochées : $1596\left(\mathrm{n}^{\circ} 13\right), 1608\left(\mathrm{n}^{\circ} 14\right), 1618\left(\mathrm{n}^{\circ} 16\right), 1628\left(\mathrm{n}^{\circ}\right.$ 19), $1638\left(n^{\circ} 20\right), 1667\left(n^{\circ} 23\right)$. Les coutumes locales non commentées prenaient place après la coutume générale : les exemplaires conservés à la BMIU montrent que, lors de la réédition de 1628 , les coutumes locales étaient sorties un an avant la coutume générale, mais que, néanmoins, les deux parties se présentaient de la même façon et pouvaient être 
reliées ensemble. L'auteur intitule avec raison son travail Paraphrases parce qu'il prend la coutume article après article et qu'il n'ajoute souvent pas grand chose au texte, mais il sait pourtant, parfois, prendre position: à propos du chapitre 15 Des associations, il suggère de ne pas tenir compte des conventions aléatoires, ineptes, ridicules et contraires aux bonnes mœurs mais de les reconnaitre quand elles correspondent à de vraies communautés. Prohet $\left(\mathrm{n}^{\circ} 25\right)$ affirme que Basmaison était très intelligent en droit coutumier et qu'il est dommage qu'il ne soit pas passé de ses paraphrases au commentaire qu'il avait promis ${ }^{32}$.

Pour l'édition de 1667, qui intervient soixante-dix ans après la première édition et trente ans après la dernière, Guillaume Consul a modernisé le français qui datait de 1596, ajouté en marge soit des explications, soit des références à des jurisconsultes modernes, à Cujas en particulier, à la législation royale, à la pratique et aux coutumes voisines, proposé enfin des rapprochements entre articles y compris avec les coutumes locales, mais il n'a pas cherché à renouveler foncièrement le texte de Basmaison et surtout, comme son livre est paru précisément en 1667, il n'a pas pu intégrer la nouvelle ordonnance civile et encore moins celles qui l'ont suivie. C'est peut-être la raison pour laquelle sa version n'a pas été rééditée.

Il y a ensuite comme un vide et c'est en 1695 seulement qu'apparaît un nouveau commentateur Claude-Ignace Prohet. C'est lui désormais qui sera réédité. Comme Jean de Basmaison, il se réclame de du Moulin mais il ne cite pas son prédécesseur immédiat, donnant ainsi son travail comme une entreprise nouvelle. Accentuant, en effet, l'évolution constatée chez Guillaume Consul, il cite parfois le droit civil mais beaucoup plus souvent les autres coutumes de la région, des traités de juristes écrivant en français et la législation royale, en particulier les grandes ordonnances de la première partie du règne de Louis XIV qui ont rationalisé et uniformisé la procédure en dérogeant aux coutumes. Cette première édition est parisienne mais c'est la suivante, celle qui est paru en deux tomes à Clermont-Ferrand en 1745 qui est la plus représentée à la BNF.

Dans cette édition, les Observations de Prohet, sont augmentées de notes qui concernent surtout les articles qui ont été abrogés ou changés par les nouvelles ordonnances de Louis XV par Mr. ${ }^{* * *}$ avocat, dont le nom Artaud, précisé par une mention manuscrite sur l'exemplaire de la BMIU, a été repris dans la notice du catalogue. Il s'agit des ordonnances dites de Daguesseau qui ont modifié les procédures en matière de testament et de donation. Sur les autres points, Artaud reprend, souvent sans le modifier, le texte de Prohet; il ajoute des exemples pris dans la jurisprudence récente aussi bien de la partie de l'Auvergne régie par le droit écrit que dans l'Auvergne coutumière.

La dernière édition de Prohet date de 1770. La page de garde cite du Moulin, Prohet et Artaud sous son masque qui apparemment ne trompe personne ; c'est ensuite un avis du libraire qui sert de préface comme si aucun nouveau juriste n'avait été chargé de cette mise à jour. Sous $\mathrm{M}^{\mathrm{e} * * *}$ qui désigne Artaud, $\mathrm{c}^{\prime}$ est à la main qu'on a rajouté « et avec les peines de $\mathrm{M}^{\mathrm{e}}$ Jean-François Gaultier-Biauzat ", attribution reprise au catalogue de la BMIU. Les jurisconsultes cités dans les notes de cette édition, sont des spécialistes de droit coutumier, mais ce ne sont pas des Auvergnats.

Le dernier commentateur de la coutume fut Guillaume-Michel Chabrol qui à fait paraître à Riom entre 1784 et 1787 une œuvre en quatre volumes in $-4^{\circ}$, totalisant 3346 pages, alors que dans leur première édition $\left(n^{\circ} 1\right)$ les coutumes ne comptaient que 250 pages in- $8^{\circ}$. L'ensemble, à douze livres le volume, coûtait quarante-huit livres alors que la dernière édition du commentaire de Prohet-Artaud en 1770 n'en coûtait que quinze ${ }^{33}$. En disant 
qu'il reprend les notes de Charles du Moulin, Toussaint Chauvelin, Julien Brodeau et JeanMarie Ricard, il indique qu'il ne reprend ni Prohet ni Basmaison. En parlant de " coutumes générales et locales de la province d'Auvergne ", il se permet de changer un titre qui était resté le même pendant deux siècles et demi. Il change aussi l'usage qui était d'appeler «chapitre » et non "titre » chacune des trente et une grandes parties de la coutume. Dans deux cas il en a aussi modifié le titre : en tête du chapitre 12, au lieu de "successions et testaments", il a mis "successions testamentaires et dispositions de dernières volontés ». En un sens il a raison, la coutume ne parle pas des successions $a b$ intestat mais uniquement des successions testamentaires, mais il a tort de changer un texte officiel. Enfin il a très gravement changé le texte et l'esprit même des coutumes locales en les présentant par ordre alphabétique et non dans le cadre des entités féodales et dans un ordre approximativement géographique, comme elles l'étaient jusque-là.

L'ouvrage débute par deux Dissertations historiques, l'une sur l'origine de l'introduction du droit écrit et du droit coutumier dans la province, l'autre sur la forme dans laquelle la justice a été rendue en Auvergne depuis l'établissement de la monarchie. Avec leurs nombreuses annexes, ces deux dissertations qui ne sont pas sans rapport avec les coutumes mais qui ne sont pas indispensables à leur commentaire, occupent cent quatrevingt dix-huit pages. Chabrol y fait preuve de plus d'érudition que de sens de la formule historique. Le commentaire proprement dit est absolument exhaustif: la législation royale, Masuer, la jurisprudence, les jurisconsultes, les autres coutumes, le droit romain très peu, les prédécesseurs de Chabrol parfois, ne serait-ce que pour les critiquer, sont cités dans le texte mais rarement référencés en marge. Même quand Chabrol signale d'emblée qu'une ordonnance a rendu caduc un article, il ne peut s'empêcher ensuite de transcrire les fiches qu'il a rassemblées à son sujet. À tout propos, on a droit à l'explication du sens des mots par l'usage ou par l'étymologie.

La jurisprudence citée remonte parfois à la première moitié $\mathrm{du} \mathrm{XVI}{ }^{\mathrm{e}}$ siècle et ne descend guère plus bas que 1750 , elle provient de recueils d'arrêts et non de l'expérience de l'auteur qui a pourtant été jusqu'en 1780 avocat du roi au présidial de Riom; il sort très facilement du cadre auvergnat et même du parlement de Paris alors qu'il semble parfois mal au courant de la pratique des notaires ruraux. Dans les meilleurs développements historiques, ceux dans lesquels Chabrol nous montre que le droit évolue, il ne s'appuie pas sur la jurisprudence mais sur les positions successives de ses prédécesseurs censées représenter la jurisprudence de leur époque : c'est le cas par exemple quand, dans le commentaire du chapitre 11, il parle de l'émancipation par mariage; mais comme Chabrol ne veut jamais renoncer à faire état de tout ce qu'il connait, il cite aussi des arrêts pris cas par cas, si bien que l'érudition juxtaposée à l'exposé menace d'en masquer le sens. Le poids de l'érudition est encore plus visible dans le tome consacré aux coutumes locales : comme il est bien difficile de trouver quelque chose à en dire surtout si on les prend une à une, Chabrol raconte au sujet de chaque juridiction l'histoire des familles seigneuriales qui l'ont tour à tour possédée. L'histoire que nous propose Chabrol repose sur des bases documentaires beaucoup plus sûres que celle de Savaron mais elle se noie dans son érudition.

Comme la Révolution est intervenue deux ans plus tard, le livre n'a jamais été réédité. On ne peut pas savoir ce qu'aurait été son destin si le droit coutumier persistant lui avait conservé sa raison d'être ni si la postérité l'aurait considéré comme un livre de jurisprudence ou comme un livre d'histoire. 


\section{Le goût de l'histoire}

77 Dans le corpus limité que constitue ces publications des coutumes, on constate que le goût pour l'histoire érudite ne date pas de Chabrol. En 1740 ( $\left.\mathrm{n}^{\circ} 26\right)$, un éditeur clermontois avait donné une édition de la coutume seule, ce qui n'était pas arrivé depuis des générations : l'intérêt de ce livre, si on en croit le titre, résidait dans le respect de l'orthographe d'origine comme si ce volume était destiné à figurer dans les bibliothèques plus au rayon histoire qu'au rayon droit.

Alors qu'en 1667 ( $n^{\circ} 23$ ) Consul modernise le vocabulaire de Basmaison sans le signaler, une génération plus tard, en 1695 ( $\left.n^{\circ} 25\right)$, Prohet éprouve le besoin pour présenter son travail de faire l'histoire des commentaires précédents ; il s'intéresse à l'origine du droit écrit : avant Chabrol, il a vu que celui-ci est observé dans les villes «qui dépendent des évêchez et des autres bénéfices de la province "; commençant sa paraphrase par le titre traditionnel des coutumes et tombant sur le mot "Auvergne ", il résume l'histoire de la province; enfin il s'engage dans le long sous-titre de son livre à donner l'histoire des familles nobles et des fondations religieuses. Il tient sa promesse dans le cadre de chaque coutume locale de façon moins détaillée que Chabrol un siècle plus tard mais sans davantage de rapport avec les articles qu'il est censé paraphraser. Lui aussi a de la documentation à placer parce que, associé aux recherches de noblesse menées par l'administration royale, il a eu l'occasion de consulter les dossiers de toutes les familles seigneuriales.

Le sens du document historique fait qu'au bout de deux siècles et demi d'éditions qui se recopiaient l'une l'autre, le libraire-éditeur de $1770\left(\mathrm{n}^{\circ} 28\right)$ se vante d'avoir fait relire le texte qu'il donne sur la copie exacte qui se trouve au greffe du parlement de Paris; Chabrol dit s'être livré à la même opération sur l'original qui se trouve au greffe de la sénéchaussée d'Auvergne.

À côté des éditions des coutumes, on trouve à la BMIU, éditées au XVIII ${ }^{\mathrm{e}}$ siècle, des opuscules de droit auvergnat qui s'appuient sur l'histoire. En 1748 un certain Tixier avocat commence son Discours sur l'origine du partage de l'Auvergne en pays de droit écrit et en pays de droit coutumier ; " en écrivant on désire naturellement connaître la loi de son pays mais il est rare qu'on veuille remonter à ses origines et poursuit en donnant un travail d'histoire locale érudit ou se voulant tel $\aleph^{34}$. Il est normal dans l'esprit du droit d'Ancien Régime que tout mémoire visant à établir le droit d'un plaideur cherche à faire la preuve de son ancienneté : c'est ce qu'on trouve dans les libelles qu'échangent les sénéchaussées de Riom et de Clermont sur leurs ressorts respectifs; c'est ce que fait Chabrol quand il défend son droit de triage contre les habitants de ses seigneuries, mais ce qui est nouveau, ce sont les ouvrages qui font état gratuitement de recherches de documents anciens. En nombre de pages imprimées, le droit continue pourtant à l'emporter sur l'histoire grâce aux éditions des coutumes.

\section{L'importance du droit}

81 C'est pourquoi il faut remettre en cause l'impression d'une désaffection vis-à-vis des coutumes, désaffection que traduirait l'espacement des éditions commentées.

Si nous laissons de côté les trois éditions de commentaires qui prennent place au cœur de la période où on édite le plus souvent les coutumes seules, qui ont lieu hors de Clermont, 
en latin et qui concernent des ouvrages dont les auteurs sont très ignorants du droit auvergnat ou peu familiers avec lui, nous avons par la suite trouvé de 1596 à 1640 six éditions en moins de cinquante ans dont cinq très proches de la jurisprudence auvergnate, puis de 1640 à la Révolution, six en cent cinquante ans, dont quatre dans le dernier siècle et trois dans le dernier demi-siècle, ce qui traduit à nouveau une vraie demande.

Il serait impensable en effet que l'intérêt se soit détourné de la coutume qui restait le code de la province : les actes de la pratique montrent à chaque instant que les coutumes sont appliquées, et les brochures imprimées pendant la période du droit intermédiaire montrent à quel point il fallait, à ce moment-là encore, compter avec elles. Il n'y aurait aucune raison, de fait, pour qu'il en soit autrement.

Si les éditions s'espacent parfois, c'est que le nombre d'exemplaires disponibles dans le public lui suffisait. Pendant quarante ans, on réédite Basmaison tous les dix ans parce qu'il était le premier commentateur proche des réalités auvergnates; le livre de Prohet ne suscite pas le même engouement, parce qu'on continue à utiliser Basmaison après la parution de Prohet même si celui-ci, paru un siècle après Basmaison, apporte du nouveau. Les anciennes éditions en effet restent dans le circuit. L'exemplaire de première édition des coutumes $\left(n^{\circ} 1\right)$ qui est conservé à la BMIU, a été relié avec une soixantaine de feuillets non imprimés couverts de notes de plusieurs écritures manuscrites qui s'échelonnent $\mathrm{du} \mathrm{XVI}{ }^{\mathrm{e}}$ au XVIII ${ }^{\mathrm{e}}$ siècle ; sur les exemplaires d'autres éditions, ce sont les $e x$ libris qui se succèdent; d'autres encore ont été à un certain moment dotés d'une reliure neuve qui insère une page blanche à la place d'une page perdue. C'est pourquoi chaque édition grossit le nombre des exemplaires en circulation. En 1587 au bout de sept éditions, on peut supposer qu'il y en avait plus de mille.

Certains de ces exemplaires sont à Paris entre les mains de conseillers au Parlement pour les aider à juger les causes venues en appel d'Auvergne, mais aucune bibliothèque de parlementaire parisien n'a jamais contenu toutes les coutumes de cet immense ressort: les grands coutumiers à partir du début du XVII ${ }^{e}$ siècle seront faits pour répondre en un seul livre à ce besoin. Les avocats, auxquels les Auvergnats ont l'habitude de s'adresser quand ils ont un procès au Parlement, possèdent plus sûrement que les magistrats un exemplaire des coutumes mais ils ne doivent pas être très nombreux et ces volumes se transmettent de génération en génération. Les autres exemplaires sont en Auvergne puisque, dès le premier tirage parisien, il était prévu de vendre la coutume à Clermont.

Dans les tables de localités qui accompagnent à partir de 1608 la publication des coutumes locales, on trouve neuf cent trente et un toponymes pour cent quatre-vingt juridictions ${ }^{35}$. Avec mille exemplaires fournis en soixante-quinze ans par les sept premières éditions, l'Auvergne en avait momentanément sans doute assez. L'édition suivante des coutumes seules, celle de 1627 n'intervient que quarante ans plus tard. À partir de 1596, on a, en revanche, vendu beaucoup de commentaires.

Pas plus que la survie des livres, il ne faut sous-estimer le rôle des copies manuscrites à usage personnel: la bibliothèque de Clermont-Ferrand conserve le manuscrit d'un commentaire qui n'a jamais été publié et que les conservateurs, au vu de l'écriture, ont daté de $1720^{36}$; parce qu'il emploie des tournures archaïques, qu'il mêle le latin au français et que l'auteur donne parfois même l'impression de penser en latin, il est vraisemblable qu'il a été composé plus tôt; il s'est pourtant trouvé quelqu'un pour le copier au début du règne de Louis $\mathrm{XV}$. 

1745 ( $n^{\circ}$ 27) l'Avis du libraire explique que "la disette extrême des précédentes éditions rendait la lecture de cette loi moins facile et moins familière aux habitants de cette province, et que la rareté de ses exemplaires privait le public des avantages que doivent lui procurer l'étude et la connaissance d'une loi de laquelle dépendent les droits des particuliers, l'intérêt et la fortune des familles ». Comme en $1770\left(\mathrm{n}^{\circ} 28\right)$, en tête de l'édition suivante, le même éditeur, Pierre Viallanes expose que «toute la province d'Auvergne sait que la dernière édition de ses coutumes avec notes et commentaires fut épuisée en peu de temps »: c'est la constatation qu'il existe une demande qui détermine un libraire à procéder à une nouvelle édition. au $\mathrm{XVII}^{\mathrm{e}}$ siècle crise des vocations. Lorsqu'il arrive au barreau, il entend les anciens se plaindre de ce qu'il n'y avait pas de commentaires sur la coutume parce que Basmaison n'aurait laissé que des paraphrases. Il voit les anciens solliciter $\mathrm{M}^{\mathrm{e}}$ Gilbert Marie ancien avocat, son beau-père d'entreprendre cet ouvrage dont ils le jugeaient très capable. C'est alors que par ordre de son beau-père et sous sa direction, Prohet commence, dès 1652, à faire des observations sur les articles de la coutume d'Auvergne, à examiner ce que les coutumes voisines avaient de semblable et à apprendre les interprétations que les arrêts et les sentences avaient fait des difficultés qui s'étaient présentées. Il publie quarante ans plus tard alors qu'il doit avoir plus de soixante ans et qu'au bout d'une vie passée à accumuler des matériaux, il est à son tour un "ancien avocat $»^{37}$. Si préparer un commentaire demande une vie et si tous les avocats ne s'en sentent pas capables, on comprend qu'il ait pu y avoir un vide entre Basmaison et Prohet.

En 1745, il ne s'agissait pas seulement de mettre sur le marché de nouveaux exemplaires des commentaires mais de tenir compte des changements apportés à différentes dispositions de la coutume par les nouvelles ordonnances. Le libraire demande à des personnes soucieuses comme lui du bien public, des observations sur ces changements. $\mathrm{M}$ ${ }^{\text {e }}$ Artaud qui avait été chargé de cette mise à jour, avait sans doute considéré qu'il en faudrait ultérieurement une autre, car il avait accumulé et laissé de nombreuses notes manuscrites qui furent utilisées pour l'édition de 1770. Derrière l'espacement des éditions, il y a le travail continu d'une généalogie intellectuelle d'hommes de loi.

es seize ouvrages imprimés à Clermont au $\mathrm{XVI}^{\mathrm{e}}$ siècle, il y avait eu quatre éditions de la coutume seule et déjà un commentaire. Parmi les autres ouvrages on trouve un Train de pratique, un Stil, un recueil d'ordonnances, deux statuts épiscopaux, six édits ou déclarations du roi, deux récits d'épisodes des guerres civiles, un missel, une controverse relative aux conciles et un livre de poésie. Seules les coutumes ont donné lieu à des volumes de plus de cent feuillets. Éditer les coutumes, c'est un travail de même nature que d'éditer des ordonnances ou des édits royaux. Les commenter, même si c'est simplement pour les paraphraser comme l'a fait Basmaison, demande un autre type de travail : il ne s'agit pas seulement d'éviter les fautes de copies mais de réfléchir sur un texte et de rédiger le résultat de ses réflexions. La rédaction du Train de Pratique et celle du Stil de la cour des aydes ont demandé, elles aussi, un vrai travail de composition car à la base il n'y a pas de texte officiel mais seulement une série d'usages. Seul le Discours sur la tenue des conciles a pu demander un effort de conceptualisation supérieur.

$92 \mathrm{Au} \mathrm{XVII}{ }^{\mathrm{e}}$ siècle, les éditions clermontoises des coutumes ne représentent plus que neuf sur cent soixante-seize livres. Les premières rééditions de Basmaison ne sont que des reprises du travail de 1596 ; en revanche, celle dans laquelle intervient Consul, la traduction très 
remaniée de Bessian et d'Aymon et la première édition de Prohet qui, même si elle paraitt à Paris, a été évidemment préparée à Clermont, supposent une réelle application intellectuelle. C'est peu mais les cent soixante-sept autres livres ne supposent pas tous une élaboration très réfléchie: on trouve deux pamphlets politiques, soixante et onze textes législatifs ou réglementaires dont l'abondance est liée à la tenue des Grands Jours, seize œuvres littéraires dont une méthode pour rectifier la prononciation des Auvergnats, dix livres d'histoire, un traité de technologie, deux de médecine, cinq de philosophie et soixante-cinq textes religieux parmi lesquels il y a les bréviaires qui se répètent, les vies de saints anecdotiques mais aussi de gros livres de théologie.

93 Le droit n'est donc pas la seule source de réflexion, mais dans la mesure où les commentaires de la coutume reposent en grande partie sur la jurisprudence, on peut considérer qu'ils sont l'œuvre collective des hommes de loi clermontois qui en parlent entre eux comme le montrent les souvenirs de Prohet. L'histoire tourne à l'érudition, les coutumes restent les seules publications qui donnent lieu, dans la dédicace ou dans la préface, à des protestations de patriotisme local.

\section{Conclusion}

On ne peut pas conclure que l'intérêt pour les coutumes diminue lorsque les éditions s'espacent : d'une part, les livres imprimés ne sont qu'un des vecteurs d'une transmission et d'une réflexion qui se fait très souvent oralement, par exemple, en suivant les audiences, mais qui peut aussi recourir à des textes écrits à la main parmi lesquels on trouve aussi bien des arrêts, des articles de coutumes, des passages de livres et parfois même des ouvrages entiers qui n'ont jamais été imprimés; d'autre part, à partir du moment où un livre est édité, il reste en circulation très longtemps, on le fait relier à nouveau quand il se disloque, on ne renonce pas à s'en servir sous prétexte qu'un livre plus récent est paru, on le prête et on l'emprunte. On a pourtant à de nombreuses reprises, même si la distribution au cours des trois siècles fut irrégulière, édité la coutume soit seule soit accompagnée de commentaires et dans ce cas, il s'agissait de gros livres.

Même si on estime que, dans chaque juridiction, un praticien ou un juge devrait disposer d'un exemplaire de la coutume, la fréquence des éditions pendant les deux premières générations qui en suivent la rédaction officielle, fait qu'avant la fin $d u \mathrm{xvI}^{\mathrm{e}}$ siècle, ce besoin pouvait être couvert. À ce moment, en effet, les éditions de la coutume seule deviennent plus rares. C'est alors que se propage un autre type d'ouvrage et avec lui un autre besoin : la coutume accompagnée d'une paraphrase faite en français par un homme de loi local, ouvrage, dont les nombreuses rééditions, au début du XVII ${ }^{\mathrm{e}}$ siècle, témoignent presque d'un engouement du public. Au bout de quelques éditions, ce besoin semble à son tour provisoirement satisfait. L'apparition, un siècle plus tard, d'un nouveau commentaire, et le souci exprimé par le libraire lors des rééditions suivantes de pallier une pénurie traduisent sans doute l'augmentation de la population et l'élargissement du lectorat au XVIII ${ }^{\mathrm{e}}$ siècle.

96 Chacune de ces éditions ne répond pas seulement au besoin du public de pouvoir se procurer ces livres, elle révèle aussi un souci de perfectionner le produit dans sa forme, dans son contenu et dans son mode de consultation. L'usage par les éditeurs de polices variées, d'éléments de décoration, de recherche dans la mise en page et de normalisation de la présentation n'est propre ni à l'Auvergne ni aux éditions des coutumes : Clermont 
suit Lyon et Paris sans trop de retard. L'apparition des commentaires est l'élément le plus immédiatement visible de l'amélioration du contenu, mais ces commentaires eux-mêmes progressent : on passe de la paraphrase au commentaire, d'une attention inutile au droit romain et à des jurisconsultes fameux, à un enracinement beaucoup plus utile dans le droit français et dans le contexte coutumier et à une prise en compte de la législation royale dans la mesure où celle-ci déroge aux coutumes. Ce pragmatisme ne peut que retenir l'attention des lecteurs. Ces livres, enfin, améliorent les instruments de consultation qu'ils mettent à la disposition du lecteur: table des matières et table des localités. Certains de leurs lecteurs ont appris dans d'autres livres à manier un index mais d'autres découvrent dans les livres de droit ce type de rationalisation.

On ne pourrait pas déduire de notre petit corpus l'importance de la culture juridique dans la France d'Ancien Régime et il n'était pas question dans ce cadre, alors que nous n'avons même pas pu utiliser le Répertoire des livres édités en Auvergne au XviII ${ }^{\mathrm{e}}$ siècle, de comparer sa place à celle des autres formes de culture écrite. De la religion en particulier nous ne pouvons rien dire si ce n'est que la seule édition des coutumes qui lui fasse une place dans son iconographie et ses devises date de 1657. On aperçoit davantage l'histoire, ce qui n'est pas étonnant compte tenu des problèmes posés par l'origine de droits pluriels, de l'importance des notions de droit acquis et d'ancienneté, de la possibilité pour les magistrats d'accéder à des documents anciens et de la place que les robins ont toujours joué en histoire. Les éditions des coutumes évoquent ou montrent à Clermont, comme semble-t-il dans bien des provinces, la triste victoire de l'érudition qui multiplie les généalogies sans doute exactes mais sans but. Les commentaires de la coutume en dépit de leur ambition d'aider à résoudre des problèmes pratiques, représentaient une nourriture plus solide et n'ont pu ainsi que contribuer à renforcer la culture juridique.

\section{Annexe 38}

\section{Gour. 300. REP. XVI e. P. 177 n $^{\circ} 1$. BMIU 98 : R 5320}

$17,5 \times 12,2$

4 ffnc. 1-95 ff. 13 ffnc.

Caractères gothiques.

Des pages non imprimées sont prises dans la reliure; elles ont servi à prendre des notes manuscrites dans lesquelles on distingue une écriture $d u x^{e} I^{e}$ et une écriture du XVIII siècle.

Page de garde :

Les coustumes du hault \& bas pays//Dauvergne

Marque de Jean Petit.

Et sont lesdictes coustumes a vendre a Paris Rue//sai(n)t Jacques a lenseigne de la fleur de liz dor. Et a Lyon//en la rue merciere deva(n)t saint Anthoine : en la boutique// de Jehan petit. Et a Clermont en l'hostel de Loys Mari//tain libraire dud(it) lieu.

Texte du privilège.

Deux lettres dédicaces.

Table des chapitres.

Titre courant avec une erreur à partir du folio 88 .

Colophon : 
Cy finissent les coustumes dauvergne//imprimées a Paris le.VIII. jour de may.//mil. CCCCC. et. XI. pour Jehan petit ://libraire de l'université dudit lieu : avec le pri//vilege de la court de parlement: laquelle a//ordonne que nul autre que ledit petit ne puisse imprimer ne faire imprimer lesdictes//coustumes de deux ans : comme il appert//par le double dud privilege insere au $\mathrm{p}(\mathrm{re}) \mathrm{mier} / /$ cayer de ce present livre.

Second texte sous la même reliure et destiné dès l'origine à accompagner le premier : il ne lui est postérieur que de quelques mois, son titre le présente comme une suite et il est compris dans le même privilège.

3 ffnc.

Page de garde :

Sensuyvent les lectres patentes//du roy nostre sire octroyées sur la reformation//des poix : mesures et aulnages du hault et bas pays Dauvergne.

Marque de Jean Petit.

Et sont lesdictes ordonnances a vendre a//Paris Rue//saint Jacques a lenseigne de la// fleur de liz dor en lhostel de Jehan petit.

Colophon :

Imprime a Paris le vingtième jour de may mil CCCCCXI pour Jehan petit libraire de luniversite dudit lieu: avec le privilège de non les imprimer de deux ans: comme il appert par le coustumier dud. pays.

\section{Gour. Non. REP. $x{ }^{e}{ }^{e}$ p. $177, n^{\circ} 2$. BMIU 99 : R 5322}

Caractères gothiques.

$14,5 \times 10,2$

8 ffnc. 1-94 ff. 22 ffnc.

Tout est très semblable en plus petit à l'exemplaire précédent y compris les caractères employés et la marque. Le titre et les adresses en bas sont les mêmes mais ne sont pas coupées aux mêmes endroits; des petites différences de graphie, par exemple dans le titre du chapitre 24 vente écrit en toutes lettres dans le $n^{\circ} 1$ est écrit en abrégé dans le $n^{\circ}$ 2 ; fautes d'inattention, par exemple le chapitre 27 qui dans le $\mathrm{n}^{\circ} 1$, dans le manuscrit authentique et partout ailleurs s'appelle de la condition de main morte, dans le $n^{\circ} 2$ s'appelle de la réduction de main morte.

Trois feuillets blancs au début.

Texte du privilège.

Deux lettres dédicaces.

Table des chapitres.

Titre courant : l'erreur se prolonge moins.

Colophon:

Cy finissent les coustumes dauvergne $\mathrm{i}(\mathrm{m})$ primées a//Paris le XxxI jour de septembre (sic) Mil CCCCC et XI//pour jehan petit : libraire de luniversite dud lieu avec//le privilege de la cour du parleme(n) $t:$ laquelle a ordon//ne que nul autre que ledit petit ne puisse imprimer ne//faire imprimer lesdictes costumes de deux ans : $\operatorname{co}(\mathrm{m}) / / \mathrm{me}$ il appert par le double dudit privilège insere $\mathrm{au} / /$ premier cayer de ce présent livre.

\section{Gour. 301. REP. XVI (Lyon). BMIU non ${ }^{39}$}

3 ffnc. 1-85 ff. 11 ffnc. 40 ffnc. 
Page de garde :

Les grandes coustumes general//les du hault et bas pays dauver//gne nouvelleme (n)t corrigees avec//plusieurs additions sur certains//articles et ung extraict du proces verbal//sur le dernier article des apchas, lotz et ve(n)//tes, et sur plusieurs aultres articles conten//ues audict extraict : que devant navoit este imprime audict coustumier.

Les ordonna(n)ces Royaulx faictes sur le//faict des aydes et tailles publiées et enre=//gistrees en la court des aydes à Paris En// s'ensuyvant le vouloir et ma (n)deme(n)t du Roy//nostre souverain seigneur.

Marque de Jacques Mareschal avec la devise: fais, ce. que devras. advienne, que pourra.

Texte du privilège de trois ans accordé par François $\mathrm{I}^{\mathrm{er}}$ en considération des grands frais que l'éditeur a engagé pour faire corriger le texte préalablement publié des coutumes et y adjoindre les ordonnances sur le fait des aides et tailles.

Deux lettres préfaces.

Table des chapitres avec l'adresse au bas :

Et sont lesdictes coustumes à vendre a lyon//en la maison de Jaques mareschal al's rola(nd) près//nostre dame de confort, et a clermont en la bou=//tique audict Jaques mareschal deva(n)t nostre da=//me de grace.

colophon avant les ordonnances fiscales :

Cy finissent les grandes coustumes des//bas et hault pays Dauvergne : avec les addi=//tions : et les Ordonnances royaux faictes sur //le fait des aides \& tailles publiées en la court//des aydes a Paris : nouvellement imprimees//a Clermont par Jaques mareschal $\mathrm{i}(\mathrm{m})$ primeur//et libraire devant nostre dame de grace. Et fuurent achevées le dernier jour Davril. Mil//cinq cens. XXIII.

\section{Le Répertoire p. $181 n^{\circ} 1$, assimile cette édition au $n^{\circ} 302$ de Gouron}

Le titre n'est pas le même mais il est très vraisemblable qu'il s'agisse de la même édition dont seule la page de garde changerait selon que l'éditeur s'adresse à la clientèle de Clermont ou à celle de Lyon.

Les Coustumes du hault et bas pays d'Auvergne, nouvellement imprimées, veues et corrigées. Et sont les dictes Coustumes à vendre à Lyon en rue Mercière en la boutique de Nicolas Petit devant le maillet dargent. Et à Clermont à l'imprimerie. Clermont, Nicolas Petit, 1538. In $8^{\circ}$, car. goth. 4 ffnc., $80 \mathrm{ff}$., $12 \mathrm{ffnc}$.

A la fin : Imprimées à Clermont par Nicolas Petit l'an de grâce 1538 et le 28e jour de mars.

4 bis. gour. 302. rep. $x V I^{e}$ p. $181, n^{\circ} 1$. bmiu 100 ; r 5324

$16,6 \times 10,1$.

6 ffnc. 1-80 ff. 9 ffnc.

Caractères gothiques et caractères romains.

Page de garde bien construite :

(fleuron) LES COV = //STUMES DV HAULT ET BAS// pays Dauuergne nouvellement $\mathrm{im} / /$ primees veues \& corrigees

(fleuron) 
1538

Et sont lesdictes coustumes a vendre a Clermo (n) $t / /$ en la boutique de Jehan Durand devant la croix//Nostre dame de grace.

Deux lettres préfaces.

Table des chapitres.

Une lettre à Antoine Duprat dans laquelle la rédaction des coutumes est comparée à l'œuvre de Justinien.

Colophon :

cy finissent les coustumes dauvergne im//primees a Clermo(n)t par Nicolas Petit. la (n) de grace.Mccccc.XXXVIII. et le. XXVIII jour de Mars.

\section{Gour.303. BMIU non. REP. (Lyon)}

Caractères romains.

Titre :

S'ensuyt le tiltre d'assiette de rente, auquel conséquemment sont adjoustées les Coustumes locales du hault et bas pays d'Auvergne : ensemble les noms des villes, chastellenyes, seigneuries et juridictions. Lyon, Antoine Vincent, 1548. In $8^{\circ}$.

\section{Gour. 321 . BMIU $103 ; 30016$}

$16,7 \times 10,2 \times 3,5 \mathrm{~cm}$.

$12 \mathrm{ffnc}$. 1-248 ff. 1-47 ff.

Page de garde :

ARVERNORUM//CONSVETVDINES//Ioannis Bessiani a Pressaco Iuris-//consulti clarissimi olim apud Montem-//ferrandum in Aruernis, nunc in senatu//Tholosano causarum patroni An-//notationes locupletissimee atq(ue) //etiam doctissime in Aruer//norum co (n)suetudines ://Opus recens natu(m)//ac nu(n)c primum//in lucem e-//ditum.

Adectus est Index copiosissimus rerum//omnium memorabilium quae in hisce// annotationibus habentur.

Accesit praeterea sub finem operis capitum//omnium Elenchus sermone.

LVGDVNI, //Apud Antonium Vincentium.//M.D.XLVIII.// CVM PRIVILEGIO REGIS.

Caractères romains de trois tailles ; italiques ; majuscles imagées.

Table des chapitres.

Table des matières.

Le commentaire latin est donné sur deux colonnes alors que l'article à commenter est donné en français sur toute la largeur de la page.

A la fin du chapitre 30, p. 248 :

Finis constitutionum alverniae nuper in gratiam studiosorum diligenter emendatarum et nunc primum sumptibus honesti viri Antonii Vincentii apud Dionysium Harseum Lugduni excussarum M D XLVIII

Le chapitre 31 est placé après la page de garde des coutumes locales.

Sensuyt le titre d'Assiette de rente, di//stingue par Articles, auquel conse-// quemment sont adioutes les cou//stumes locales du bas pays//d'Auvergne, ensemble les//no(m)s des villes, Chastel//lnyes, seigneuries//\& iuridi//ctions.

A partir de là il n'y a plus de commentaire, donc plus de latin, plus de colonnes. 


\section{Gour. 311. BMIU non}

Commentarii in Consuetudines Arvernie editi par D. Aymonem Publitium pedemontamum, clarissimum juris utriusque doctorem ac olim Allobrogum praesidem, summaris ac indice alphabetico illustrati. Parisiis, apud Poncetum Lepreux, 1548.

8. gour. 312. bmiu $105 ; 1037$

$32,0 \times 21,3$

$10 \mathrm{ffnc}$. $1-173 \mathrm{ff}$.

Page de garde :

COMMENTARII//in Consuetudines Arver-//NIAE, EDITI PER D. AYMONEM//Publitium Pedemontanum, clarissimum Iuris//utriusqu e doctorem, ac olim Allobro-//gum Praesidem, sunmarii, ac indi-//ce alphabetico illustrati.

Doce me facere uoluntatem tuam.//Psal. 142.

Marque de Poncet Le Preux.

Cum priuilegio Senatus.//PARISIIS, Apud Poncetum le Preux, in via S. Iacobi, sub signo Lupi, e regione Matbrinorum.// 1549

Texte du privilège du livre.

Dédicace à Jean Bertrand, docteur en droit.

Lettre à Antoine Dulac, docteur en droit.

Index titularum

Index alphabeticus eorum que continentur in his commentariis

Deuxième titre au début de la coutume quand la foliation commence :

GLOSAE NE DIXERIM UTILES, IMO//ET NECESSARIAE EDITAE AC COMPOSITAE//super consuetudinibus Alverniae per Do. Aymonem, ex comitibus//Plozaschi et publitiarum condominus Pedemontanum, iuris utiusque//doctorem clarisssimum et olim Sabaudiae proesidium.

Son livre ne contient pas les coutumes locales et certains articles du dernier chapitre de la coutume générale ont été omis.

Il cite les articles en français puis il les traduit en latin.

\section{Gour. 304. REP. $x I^{e}$ p. $182, n^{\circ} 3$. BMIU $101 ; 5325$}

$11,3 \times 7,5$

2 pp. I-CCCXII pp. A partir de la page 53 les pages de droite sont paires.

Page de garde :

bien construite; en bas de page, le lieu, l'éditeur et la date sont disposés de la façon qui deviendra classique.

Les Coustumes//Du Hault \& Bas pays d'Auuer-//gne nouuellement Impri-//mees, reuues,//corrigées//oultre les precedentes//Impressions.

Plus de nouueau y est adiouste//le Train de praticque.

A CLERMONT//Chez Iean Durand demeurant//pres nostreDame de Grace.

1552

Table des chapitres.

Titre courant avec l'erreur de l'édition $n^{\circ} 1$.

Il n'y a plus de colophon mais simplement la mention FIN.

Seconde partie de la publication. 
Page de garde :

LE// Train de Pra-//CTIQUE, ov L'OR-//dre iudicier contenant la for-//me de proceder en tout-//tes les instances de//iugement : ne-//cessaire à//tous//Practiciens.

Plus bas :

si de plaider tu as ennuie,//Regarde, ou, quoy, quand \& comment,//en ton plaict ne fais faulx comment//en faicts \& dictz gist mort, \& vie.

En bas :

A CLERMONT.//Par Iean Durand.//1552.

I-IV. I-XXVI ff.

Table des chapitres.

Elle se termine comme elle a commencée par des vers de mirliton suivis du mot FIN.

\section{Gour. 305. Répertoire $x V l^{\circledR}$ p. $183, n^{\circ} 6$. BMIU non}

Les coustumes du haut et bas païs d'Auvergne, revues et corrigées avec le train de pratique, A Clermont, 1570, Jean Durand, in $8^{\circ}$.

$\mathrm{Ni}$ Gouron ni le répertoire ne donnent de détails très vraisemblablement parce que cette édition ressemble à la précédente.

\section{1. gour. non. rep. XVI (lyon) ad 63 hpb 124}

$11,5 \times 6,5$

$351 \mathrm{pp}$.

Caractères romains et italiques.

Page de garde :

Les Coustumes du//HAVT ET BAS PAYS// DAWERGNE

En bas :

Imprimé à Lyon chez Gui//chard Iullieron//1581

Table des chapitres.

Table des matières.

On a rajouté à la main Ce livre a été imprimé sous le règne d'Henri III la 7ème année de son règne.

\section{Gour. 306. REP. $x V I^{e}$ p. 183 n 9 . BMIU $109 ; 5326,35802^{40}$.}

$16,8 \times 11,2 ; 16,2 \times 10,7$. La différence vient de la reliure.

1-199 pp. Erreur à partir de la page 117.

Page de garde :

LES COVSTV//MES//DU HAVT ET BAS//PAYS D'AV-//VERGNE. Nouvellement imprimées, reuuës \& corrigees oultre les precedentes impressions.

Marque de Jean Durand

A CLERMONT//De l'Imprimerie de Iean Durand,// demeurant pres nostre Dame//de Grace.

M. D. LXXXVII.

Table des chapitres.

Une petite frise de feuillage avant le début de la coutume générale.

Une frise de grotesques avant le début des coutumes locales. 


\section{Gour. 314. REP XVI ${ }^{e}$ p. $184 n^{\circ} 17$. BMIU on}

Paraphrase de M.//Iean de Basmaison Povgnet//Advocat En la Senechavlcee//et siege presidial de Riom, Sur les Coustumes//du bas et hault pays d'Au-//vergne,//Avec Les Annotations De M. Charles Du//Moulin : et le Procez verbal au long prins sur l'original des-//dictes coustumes.

(gravure)

A Clermont,//Chez Iean Durand.//M.D.XCVI.//

Avec Privilege Du Roy.

\section{4. gour.315. bmiu 30506}

$16,0 \times 12,0$

1-6 pp. 1-683 pp. 1-61 pp.

Utilise une grande variété de caractères.

Page de garde :

(Une petite frise de feuillage)

PARAPHRASE// DE M. IEAN DE BASMAISON//Advocat au Siège Présidial de Riom sur//les coustumes du bas et hault pays//d'Auvergne,

Avec les annotations //de M. Charles du//Moulin, REVUEU DE NOUVEAU ET ILLUSTREE//de deux Tables alphabetiques tres-amples, l'une des//matieres principalles \& des villes \& villages qui se//gouvernent selon le droit ecrit ou la coustume. (fleuron).

A Clairmont par Bertrand Durand Imprimeur du Roy.

M. D C. VIII.

Avec Privilege. On a rajouté à la main jusqu'en 1608

Page de dédicace :

(Frise : oiseau et feuillage)

AUX TREZE ANCIEN-//NES ET MAISTRESSES VILLES// du bas pays d'Auuergne representans//le corps du tiers \& commun//Estat.

Une lettre préface.

Une dédicace :

A MONSIEUR VANGON ADVO//cat du Roy en la seneschaulcee et siege//presidial de Clairmont.

Table des chapitres.

La deuxième pagination est pour la coutume elle-même.

(frise : grotesques)

A la fin de la coutume générale :

(un gros fleuron : écu, animaux et feuillages)

Deuxième pagination pour les coutumes locales

(Frise : feuillage et têtes d'animaux)

S'ENSUIVENT LES//Coustumes Locales du Bas//pays d'Auvergne

Plus bas

COUTUMES LOCALES : : Des prevostez, Seigneu//ries \& chastellenies, lieux et vil-// lages du haut pays des mon-//taignes d'Auvergne

Dernière pagination pour les tables.

(Frise : oiseaux et feuillage)

Table des chapitres. 
Table des matières.

Table des localités.

À partir de maintenant je ne décris plus que les publications des coutumes seules sans commentaire. Pour les éditions commentées je ne donne que les références dans la Bibliographie et à la BMIU.

\section{1613 Rigault Gour. 314. BMIU 114, R 5328}

\section{1618 Basmaison Gour. 315. BMIU 30506}

\section{Gour. 307. REP. XVII e t. II p. 14 n $^{\circ} 13$. BMIU $106 ; 30012$}

$15,9 \times 9,6$.

1-201. 26 ppnc.

Page de garde :

LES COVSTVMES//DV BAS ET HAVT PAYS//D'AVVERGNE//ET LES PROCEZ VERBAUX AV LONG//faicts par Mess. Anthoine Duprat lors premier Président//à la Cour de Parlement et despuis Chancelier de//France, \& Loys Picot conseiller du Roy en sad.//Cour de Parlement, Commissaires ordonnez//par Sa Majesté pour la rédaction,//publication \& auctorisation,//desdictes Coustumes.

REVEVES, AVGMENTEES DE NOVVEAV//d'une Table Alphabétique des Coustumes locales des//lieux qui se régissent par le droict escrit,// ou par la Coustume.

PLUS A ESTE ADIOUSTE LES REGLEMENS// de la iustice ordonnez par Messieurs les Iuges \&//Magistrats des Seneschaucees \& Sieges Presi-//diaux de clairmont et Riom : Ensemble//les Taxes des expeditions qui se//font en icelles.

Les règlements sont prévus pour être reliés avec les coutumes puisqu'ils figurent sur le titre, ils le sont mais ils portent leur propre cote A 30013

marque: un rectangle formé d'une bordure de petits motifs floraux et d'un centre couvert de motifs plus grands.

adresse :

A CLAIRMONT,//Par Bertrand Durand, Imprimeur//ordinaire du Roy.

M. DC. XXVII.

Table des chapitres.

Table des matières.

Table alphabétique des coutumes locales.

18. Gou. 308. REP. XVII t. II p. $15 n^{\circ} 15$. BMIU 108 bis ; 30193

$22,3 \times 16,1$

135 pp.

Page de garde :

LeS CovstVMes locales dV BAS et havlt Pays d'Auuergne. Et le Procès Verbal au long desdictes Coustumes.

La marque de Jean Durand le père que Bertrand Durand le fils avait déjà utilisée dans l'édition de 1608 du commentaire de Jean de Basmaison. En 1587 et en 1608, elle était présentée dans un cadre ovale; elle est maintenant dans un cadre rectangulaire et la main verseuse vient de la droite au lieu de venir de la gauche. La devise est la même mais elle est sectionnée pour occuper les quatre côtés : DU//RANT//TEMPE//RATA. 
En bas :

A CLAIRMONT//par Bertrand Durand imprimeur du Roy.

M. D C. XXVII

Liste alphabétique des coustumes locales.

19. 1628 Basmaison Gour. 316. BMIU 30190

20. 1638 Basmaison Gour. 317. BMIU on

21. 1640 Aymon et Bessian, Gour. 313. BMIU 30192, 30193, 36658

22. 1661 Bessian Gour. 322. BMIU 30015

\section{1667 Basmaison-Consul Gour. 318 BMIU 30190}

24. Gou. non. REP. XVII ${ }^{e}$ t. II p. $24 n^{\circ} 106$. BMIU $109 ; 30190$

\section{$22,1 \times 16,3$}

1-86 pp.

Relié avec le commentaire des coutumes de Basmaison de la même année. Si je le présente à part, c'est uniquement parce que le Répertoire des livres imprimés a attribué à chacun d'eux un numéro. Le mot Fin et la grande vignette qui terminent cette partie valent pour les deux. La table des matières concerne essentiellement la coutume générale commentée.

$128 \mathrm{pp}$.

LES//COVSTVMES//LOCALES DV BAS ET//HAVT PAYS D'AVVERGNE.

ET LE PROCES VERBAL AV LONG//desdites coustumes.

Corrigés en cette dernière édition par Maître G. CONSUL Advocat en Parlement.

Marque :

Deux écus surmontées d'une couronne fleurdelisée et entourés de rinceaux et de feuillages.

Adresse :

A CLERMONT,

Par IEAN BARBIER Imprimeur ordinaire du Roy, \& marchand Libraire, proche nôtre dame de Grace.

M. DC. LXVII.

Avec Privilege du Roy.

Des frises de grotesques et des majuscules ornées en tête des différentes parties.

Table alphabétique des matières : elle renvoie presque toujours à la coutume générale commentée avec laquelle sont reliées ces coutumes locales.

Vignette de la fin :

Une couronne de laurier entoure un cercle de rayons, une devise et un IHS surmonté d'une croix. La devise dit In nomine iesu omnnes genu flectatur. 


\section{1695 prohet gour. 351. Bmiu 30191}

\section{Gou. 310. Bmiu on}

Texte de la coutume du haut et Bas Pays d'Auvergne. Imprimé sur un manuscrit original dont on a suivi l'orthographe. A Clermont-Ferrand, P. Viallanes, 1740, In-12, 355 pp., 3 ffnc.

\section{NOTES}

\section{1745 prohet gour. 352. bmiu 30037}

\section{1770 prohet gour. 353. bmiu 30194}

\section{1784 chabrol gour. 323. bmiu 30197}

1. Les calculs ont été faits à partir d'un comptage des articles dans Charles Bourdot de Richebourg, Nouveau Coutumier général ou corps des Coutumes générales et particulières de France et des provinces connues sous le nom de Gaulles, Paris, Michel Brunet, 1724, 4 vol., in-fol. Pour comparer avec la coutume d'Auvergne qui n'a pas été réformée, je me suis donné comme règle de compter les articles dans la première version des coutumes sauf dans les rares cas où la première version est une coutume médiévale dans laquelle il était difficile de distinguer les articles les uns des autres.

2. André Gouron et Odile Terrin, Bibliographie des coutumes de France. Éditions antérieures à la Révolution, Genève, Droz, 1975, 297 p.

3. Jean Masuer, Le Masuer en françoys, selon la Coustume du Hault et Bas pays d'Auvergne, et la manière comme on assist rante audit pays coustumier; et aussi les ordonnances royaux..., Imprimé à Lyon par Claude Davost alias de Troys, le dernier jour de febvrier mille cinq cens et cinq, in- $4^{\circ}, 76 \mathrm{ff}$.

4. Ce traité appelé dans sa première édition Aureus ac perutilis extractus Masuerii judiciorum praxim haud contemnendas consuetudines curieque Parlamenti supreme stilum continens... a été édité en latin huit fois à Paris, trois fois à Lyon, deux fois à Francfort-sur-le-Main et une fois à Caen; et en français sous le titre La Practique de Masuer ancien jurisconsulte et practicien de France..., six fois à Paris et cinq fois à Lyon.

5. Pour André Gouron la Practique concerne le droit auvergnat et le droit bourbonnais, mais ce n'est pas évident: c'est un livre qui peut servir partout. André Gouron «Coutumes et commentaires: essai d'analyse quantitative" in Droit privé et institutions régionales. Études historiques offertes à Jean Yver, Paris, PUF, 1976, 713 p., p. 321-332.

6. Clermont est attesté depuis 1518, Aurillac en 1520, Riom au XVII siècle; Thiers apparaît en 1518 mais disparaît rapidement in Henri-Jean Martin (dir.), Histoire de l'édition française, Paris, Fayard, 1989, t. I ${ }^{\text {er }}$. p. 188, 257 et 437.

7. Jusqu'en 1731 il faut dire «Clermont " parce que Montferrand est encore une ville distincte : c'est pourquoi la Bibliothèque municipale et interuniversitaire (BMIU) que j'ai beaucoup utilisée 
et que je citerai plus bas est celle de Clermont-Ferrand, mais que la plupart des éditions dont il sera question sont localisées à Clermont.

8. Le mot « commentaire » n'est employé, en réalité, qu'une seule fois, et encore en latin dans un des deux ouvrages parus en 1648; l'autre parle d'«annotations». En français on trouve "paraphrases ", « observations ", «notes » ou « coutumes conférées » avec d'autres formes du droit.

9. Une de nos directions de travail pour l'an prochain consistera à essayer de formaliser cette chronologie comme Martine Grinberg l'a exposé dans l'introduction générale.

10. BMIU, département patrimoine, mss 534 (A 33), $320 \times 240 \mathrm{~mm}$, parchemin, A-D et 173 feuillets, relié veau gaufré. 1510. L'exemplaire destiné au greffe du parlement pour être mis à la disposition du public devait être très semblable à celui de Clermont puisqu'ils avaient été faits parallèlement. Celui qui est conservé aux AN dans le fonds du Parlement avec son titre en lettres d'or, rouges et bleues et sa première page encadrée de feuillage or et couleur a été confectionné au moment de l'enregistrement de la coutume par le Parlement, AN X 1 $1^{\text {a }} 9218$.

11. Je remercie Dominique Frasson-Cochet conservateur du département patrimoine de la BMIU de Clermont-Ferrand d'avoir bien voulu répondre à mes inquiétudes à ce sujet.

12. André Gouron et Odile Terrin, op. cit.

13. Répertoire bibliographique des livres imprimés en France au $\mathrm{XVI}^{\mathrm{e}}$ siècle, Baden-Baden \& Bouxwiller, Valentin Koemer, 1992, t. II, p. 177-185. Répertoire bibliographique des livres imprimés en France au XVII siècle, Louis Desgraves et Jean Flouret, t. II, Baden-Baden, Valentin Koerner, 1980, 235 p., p. 12-35.

14. Jean Masuer, op. cit.

15. J'ai écrit partout Basmaison-Pougnet pour me conformer tant à la Bibliographie qu'au Répertoire mais contrairement à la Bibliographie, j'ai choisi de ne jamais mettre de majuscule aux mots «coutume» et «coutumes» parce que le plus souvent la typographie d'origine ne permet pas de trancher.

16. Je n'ai pas consulté d'exemplaires des éditions $\mathrm{n}^{\text {os }} 5,7,10,13,16,20$ et 26 . Dans ce cas, j'utilise au mieux les notices du Répertoire et de la Bibliographie. Comme la plupart de ces livres étaient en réserve ou exclus de la consultation, je n'ai eu recours à la BNF que pour consulter sur microfilm le livre de Masuer, qui n'a eu qu'une seule édition, qui ne figure pas à la bibliothèque de Clermont-Ferrand et que j'ai écarté puisque, paru cinq ans avant la rédaction de 1510, il ne peut pas reproduire le texte officiel.

17. Chacune des quatre vingt-onze coutumes locales couvre plusieurs paroisses, villages et parfois même maisons isolées qui sont citées dans son titre. La table des coutumes locales permet de savoir à propos de chaque localité - celle dans laquelle on vit ou celle d'où vient un plaideur à quelle coutume locale elle se rattache.

18. Guy de Tervarent, Attributs et symboles dans l'art profane, 1450-1600. Dictionnaire d'un langage perdu, Genève, Droz, 1958, t. I ${ }^{\text {er }}$, p. 8-9.

19. Répertoire bibliographique... XVI ${ }^{e}$ siècle, op. cit., p. $178, \mathrm{n}^{\circ} 1$.

20. Pierre Egullion, Imprimeurs, libraires et relieurs du bas-pays d'Auvergne et du Puy-de-Dôme, 1491-1939, Académie des sciences, belles-lettres et arts de Clermont-Ferrand, 2000, p. 101-102.

21. Avant de devenir avocat général au parlement de Toulouse, puis conseiller, président puis premier président au parlement de Paris, et enfin chancelier en 1515, Antoine Duprat était né à Issoire et avait été nommé en 1490 lieutenant général au bailliage de Montferrand. Son frère puis son fils furent évêques de Clermont. Il avait donc avec l'Auvergne plus de liens que n'en avaient d'ordinaire les commissaires à la rédaction avec les ressorts où ils étaient envoyés.

22. Henri-Jean Martin (dir.), op.cit., passim, voir index; Jean-Dominique Mellot et Elisabeth Queval, Répertoire d'imprimeurs libraires. XVI ${ }^{e}$-XVIII ${ }^{e}$ siècle. État en 1995 (4 000 notices), Paris, Bibliothèque nationale de France, 1997, in-4 ${ }^{\circ}, 719$ p., p. 479, notice 3017.

23. Jean-Dominique Mellot et Elisabeth Queval, op. cit., p. 417, notice 2605. 
24. La Bibliographie des coutumes cite cette édition d'après un article d'Ulysse Rouchon, «Une édition inconnue des coutumes d'Auvergne», Bulletin du bibliophile, 1923, p.98-104. Je suis retournée à cet article dont la notice de la Bibliographie ne rend pas bien compte, puisque les auteurs concluent que l'impression de cette édition a eu lieu à Lyon alors que l'article dit le contraire.

25. Répertoire des livres... XVI $I^{e}$ siècle, op. cit., t. II, p. 176-186 ; Pierre Éguillion, op. cit., p. 34-41 et 101 : l'auteur a suivi les éditeurs non seulement sur les pages de titre des livres qu'ils éditent, mais dans les minutes notariales et dans les archives de la fabrique et de la ville.

26. Jean Savaron (1550-1622) fut successivement conseiller du roi à la sénéchaussée de Riom, à la cour des aides de Montferrand et à la sénéchaussée et siège présidial de Clermont ; il est surtout connu pour avoir été le meilleur orateur du tiers état aux états généraux de 1614 dont il a également écrit l'histoire. Il était donc au-dessus du patriotisme de clocher, mais écrire «Clairmont » donnait du lustre à une des entités au nom desquelles il parlait.

27. Les engagements politiques de Jean de Basmaison qui appartient à la génération précédente, sont moins connus mais ils ressemblent à ceux de Savaron. Avocat, il est député à plusieurs reprises aux états généraux. On a gardé un de ses discours dans lequel il recommande de pardonner aux protestants rebelles de façon à ne pas irriter le mal par la dureté du remède. Comme Savaron, il eut à défendre le tiers contre les nobles. À une époque où les offices de judicature n'étaient pas encore vénaux, à la fin des états, il fut nommé lieutenant général de Clermont.

28. Entre 1570 et 1587, Jean Durand avait publié en 1579 une lettre politico-allégorique adressée au roi et en 1583 les œuvres de Salluste du Bartas. Répertoire des livres..., op. cit., t. II, p. 183, nº 7 et 8.

29. En page de garde : Si de plaider tu as envie,//Regarde, où, quoi, quand \& comment,//En ton plaict ne fais faulx comment,//En faicts \& dictz gist mort \& vie.

30. À l'époque on peut acheter un livre non relié et le faire relier à son goût.

31. Jean Besse de Preyssat.

32. Guillaume-Michel Chabrol dit dans la préface de ses Coutumes ( $\left.{ }^{\circ} 29\right)$ que Basmaison, son trisaïeul, avait préparé un commentaire en latin qui s'est perdu.

33. Ces précisions figurent sur la page de garde de l'édition de 1770 et, pour Chabrol, sur le prospectus du libraire.

34. BMIU, 95 bis, A 31520, $16 \mathrm{p}$.

35. Les paroisses ont souvent, par l'intermédiaire des collectes, donné naissance aux communes ; or il y avait huit cent cinquante-six communes dans le ressort de l'Auvergne de jadis.

36. BMIU de Clermont-Ferrand, section patrimoine, Ms 2127, c. 1720, Coutumes Du haut et bas paiis Dauvergne avec la paraphrase de M. Jean André. Chabrol connaissait deux commentaires restés manuscrits, $n^{\circ} 29$, t. I ${ }^{\text {er }}$, p. III-IV.

37. Chabrol qui est né en 1714 , publie en 1784 , à 70 ans.

38. Les références renvoient soit à la Bibliographie des coutumes (Gour.), soit au Répertoire des livres imprimés $\mathrm{XVI}^{\mathrm{e}}$ puis $\mathrm{XVII}{ }^{\mathrm{e}}$ siècle (REP) soit à la BMIU de Clermont-Ferrand : numéro dans le répertoire imprimé et numéro porté à la main dans la marge du répertoire disponible dans la salle et qui permet d'atteindre la livre ; soit dans un cas aux archives départementales du Puy-deDôme (AD 63).

39. J'ai dit plus haut que je suivais l'article d'Ulysse Rouchon plutôt que la Bibliographie des coutumes.

40. Il y a une erreur dans le catalogue imprimé de la BMIU : l'exemplaire $102=\mathrm{R} 5326$ ne mentionne pas le Train de pratique dans son titre et ne contient rien d'autre que les coutumes. L'état de conservation et la reliure ne sont pas les mêmes mais ce livre et celui qui est coté A 35802 appartiennent à la même édition. je remercie ma collègue médiéviste Josiane Teyssot 
d'avoir éclairci mes problèmes sur ce point. La différence de reliure fait que l'un est légèrement plus grand que l'autre. L'erreur a été reprise par Gouron. 